\title{
Hydration interactions: aqueous solvent effects in electric double layers*
}

\author{
Yoram Burak ${ }^{\dagger}$ and David Andelman ${ }^{\ddagger}$ \\ School of Physics and Astronomy, Raymond and Beverly Sackler Faculty of Exact Sciences, \\ Tel Aviv University, Tel Aviv 69 978, Israel
}

(5 July 2000)

\begin{abstract}
A model for ionic solutions with an attractive short-range pair interaction between the ions is presented. The short-range interaction is accounted for by adding a quadratic non-local term to the Poisson-Boltzmann free energy. The model is used to study solvent effects in a planar electric double layer. The counter-ion density is found to increase near the charged surface, as compared with the Poisson-Boltzmann theory, and to decrease at larger distances. The ion density profile is studied analytically in the case where the ion distribution near the plate is dominated only by counter-ions. Further away from the plate the density distribution can be described using a Poisson-Boltzmann theory with an effective surface charge that is smaller than the actual one.
\end{abstract}

\section{INTRODUCTION}

Electrolytes, in contact with charged surfaces or macro-ions, play an important role in determining the properties of many biological and chemical systems. One of the most widely used tools for studying ions in aqueous solutions is the Poisson-Boltzmann (PB) theory [1] 6 . The mathematical and conceptual simplicity of this theory makes it very appealing both for numerical computation [7], and for gaining insight into the underlying physical principles. Although the theory contains important simplifications, it has proven to be a useful and accurate tool in the study of systems such as colloidal dispersions [8,9], biological membranes [6], polyelectrolytes [10] and complex systems, e.g., proteins or DNA interacting with charged membranes 11 13.

The Poisson-Boltzmann theory is obtained by making two simplifying approximations. The first approximation is the treatment of the electrostatic interactions on a mean-field level. The ions are treated as independent charged particles interacting with an external electrostatic potential, derived self-consistently from the mean charge density distribution. Thus, correlations between the ion positions are not taken into account. The second approximation is the treatment of the ions as point-like objects, interacting only through the electrostatic interaction in a dielectric medium. In reality, ions in aqueous solutions have more intricate interactions [5]. These include a non-Coulombic interaction between ion pairs, which is mainly a short-range steric repulsion, interactions with the polar solvent molecules and short-range interactions with the confining charged surfaces.
Various models have been proposed for the inclusion of effects not accounted for by the PB theory. These include liquid state theory approaches 14 18, field theory expansions 19,20, computer simulations 21,23 and other modifications to the PB theory 24 27]. Most of these models remain within the framework of the socalled "primitive model", in which the interaction between the ions is modeled as a purely repulsive hardcore interaction. On the other hand, relatively few works have addressed explicitly the discrete nature of the solvent molecules 18,28 30. Clearly, the replacement of the solvent by a continuous medium cannot be precise when the inter-ion distance is comparable to the solvent molecular size. Therefore, when the ions reach high densities the discreteness of the solvent is expected to have an important effect on the ionic distribution. This is of particular importance for water. Due to its high polarity, the strong screening of the electrostatic interaction (represented by the dielectric constant) is modified at small ion separations.

Using the surface force apparatus [5], it is possible to measure precisely the force between charged mica plates. These measurements supply evidence for the importance of the solvent structure in aqueous solutions 31,32. At inter-plate separations below approximately 20 A significant deviations are found from the prediction of the Derjaguin-Landau-Verwey-Overbeek (DLVO) theory 8,33. The measured force is oscillatory or consists of a series of steps, with a period corresponding to the water molecular size. Oscillatory forces are known to arise as a result of the solvent structuring in layers between surfaces [5]. However, a repulsive contribution is found in addition to the oscillatory force at plate separations

\footnotetext{
*Accepted to Phys. Rev. E.

${ }^{\dagger}$ email: yorambu@post.tau.ac.il

${ }^{\ddagger}$ email: andelman@post.tau.ac.il
} 
below several nanometers [31,32]. This repulsive force is often referred to as the "hydration force" [5, 31], and its origin is not yet completely understood [29].

\section{A. Aqueous pair potential model}

Recently [34,35], an aqueous pair potential model has been proposed for electrolytes, in which the effect of the solvent on the ions is described as a short-range two-body interaction between the ions. The solvent is replaced by a continuum dielectric medium as in PB theory, but the ions also interact through a two-body short-range hydration interaction [34]. This is shown schematically in Fig. 1.

This aqueous pair potential model 34, 35, involves several simplifying assumptions. One is that the effect of the solvent can be represented as a linear superposition of two-body potentials between all ion pairs. Another simplification is that the effective potential between the ions is taken as the effective potential in the bulk, regardless of the ion concentration, and of the geometry imposed by the charged surfaces. Finally, a short-range surface-ion effective potential should be included in addition to the ion-ion effective potential. Despite of the simplifications made in the aqueous pair potential model, it offers a first step towards a qualitative understanding of solvent effects on the ion distribution, in particular near highly charged surfaces.

\section{B. Effective ion pair interaction}

For the short-range ion-ion interaction, the so-called potential of mean force between ions in solution can be used. Potentials of mean force are defined as $-k_{\mathrm{B}} T \log g_{i j}(\mathbf{r})$ where $g_{i j}(\mathbf{r})$ are the ion-ion radial distribution functions for ion pairs of species $i$ and $j$. The radial distribution functions have been calculated numerically for a single ion pair immersed in an aqueous solution using molecular dynamics techniques [36 38.

An alternative approach has been proposed in Refs. 39,40. In this approach, a Hamiltonian consisting of a pairwise effective potential between the ions is obtained using the so-called "reverse Monte-Carlo" approach. The ion-ion radial distribution functions are first calculated using a molecular dynamics simulation for a system including solvent molecules and a finite concentration of ions. The ion-ion effective potential in the system without the solvent is then adjusted iteratively until the same distribution functions are obtained using Monte-Carlo simulations.

The different available calculations of potentials of mean force differ in their quantitative predictions. This may be a result of high sensitivity of the models to detailed features used for the water molecules and for the inter-molecular interactions [36]. However, all the potentials of mean force as well as the effective potentials [39] are qualitatively similar [41]. Thus, for the purpose of the present work, aiming at a qualitative understanding of solvent effects, any one of these potentials may be used.

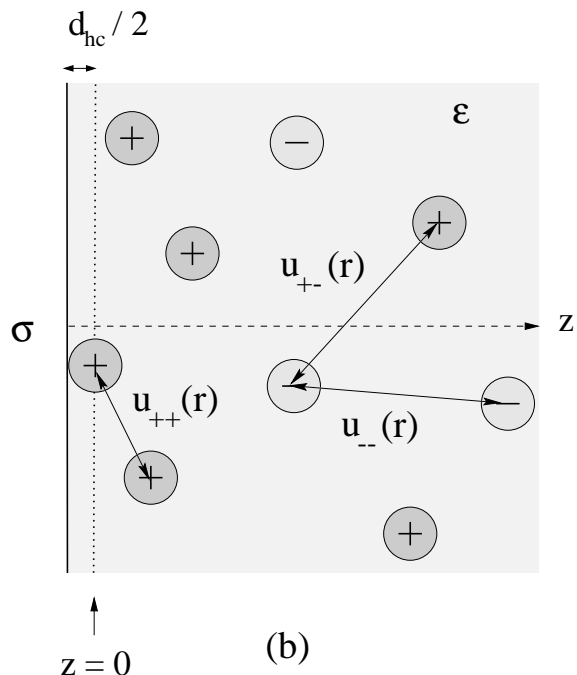

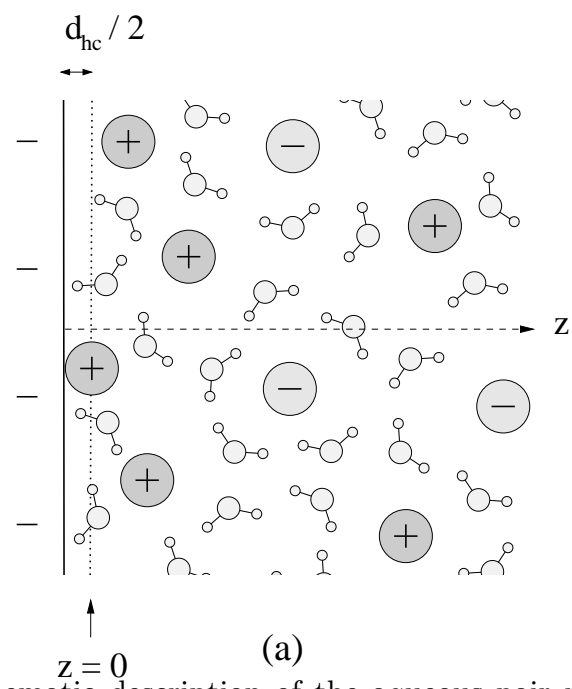

FIG. 1. Schematic description of the aqueous pair potential model. A An aqueous ionic solution in contact with a charged plate in (a) is replaced in (b) by ions in a continuum dielectric medium having a dielectric constant $\varepsilon$, with electrostatic and short-range interactions $u_{i j}(r)=u_{i j}(|\mathbf{r}|)$. The $z$ coordinate designates the distance from the charged plate, with $z=0$ corresponding to the distance of closest approach of the ions to the plate. The distance of closest approach is equal to $d_{\mathrm{hc}} / 2$, where $d_{\mathrm{hc}}$ is the hard-core diameter of the ions. 


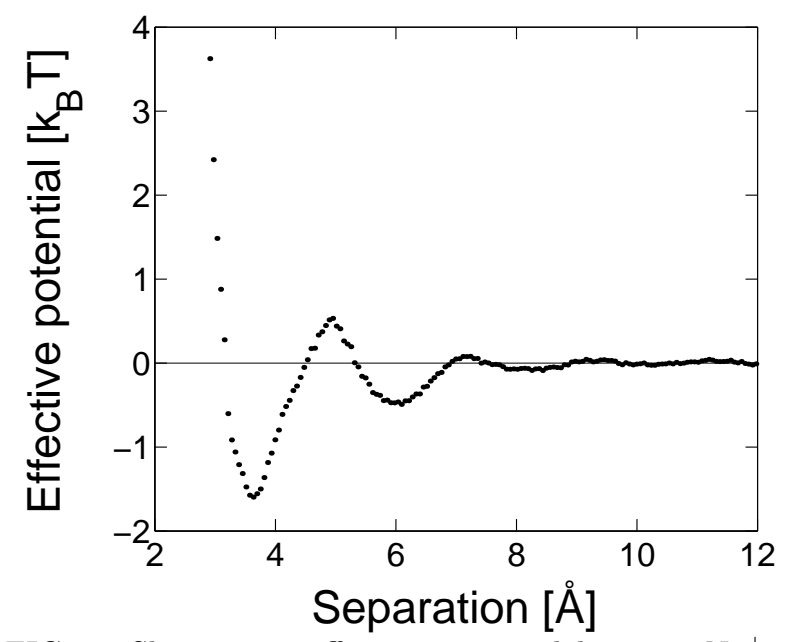

FIG. 2. Short-range effective potential between $\mathrm{Na}^{+}$ion pairs, adapted from Ref. [39] using simulations in a bulk $\mathrm{NaCl}$ solution of concentration $0.55 \mathrm{M}$, at room temperature 44. The potential is shown in units of $k_{\mathrm{B}} T$, as a function of the distance between the ion centers. For ion separations smaller than $2.9 \AA$ a hard core interaction was assumed. The Coulomb interaction is subtracted to show only the short-range hydration effect due to the water molecules.

At large ionic separations the ion-ion effective potential is well approximated by a screened electrostatic interaction, with the water dielectric constant in the continuum limit. At short ionic separation, the difference between the total effective potential and the screened electrostatic interaction is a short-range potential reflecting the structure of water molecules in the ion vicinity. Fig. 2 shows the short-range contribution (excluding the screened electrostatic part) to the effective potential calculated between $\mathrm{Na}^{+}-\mathrm{Na}^{+}$pairs in the reverse MonteCarlo approach [39]. Below about $3 \AA$, the electrostatic repulsion between the ions becomes unscreened. Therefore it is much larger than the screened repulsion in the dielectric medium, and the effective potential is strongly repulsive. The unscreened electrostatic potential leads to an effectively enlarged hard-core separation between the ions, relative to a hard-core diameter of about $2.3 \AA$ used in the short-range part of the bare ion-ion potential. At larger separations, the effective potential is oscillatory, and mainly attractive. It has a distinct minimum at an ion-ion separation of about $3.6 \AA$, followed by a maximum and a second minimum at approximately $6 \AA$.

\section{The present work}

The replacement of the discrete solvent by a continuum medium, with electrostatic and short range interactions between the ions, is a considerable simplification. Still, the statistical mechanical treatment of an electrolyte solution in this model is difficult, and requires the use of further approximations, or simulations.

The Anisotropic Hyper-Netted Chain approximation
(AHNC) 15] was previously used to calculate the effects of hydration interactions in the aqueous pair potential model 41, 42]. When the ion concentration is large enough, e.g., near a highly charged surface, the hydration interaction is found to have a significant effect on the distribution of ions in the solution. It was also proposed that the so-called repulsive "hydration forces" between surfaces arise from the ionic structure near highly charged surfaces. According to this description, at large distances from the plate, the ion distribution follows a PB profile with a reduced effective surface charge. When two plates approach each other, the ions near the surfaces come into contact giving rise to an apparent new repulsive force.

In the present work a simple description for ions interacting through electrostatic and short-range attractive interactions as mediated by the solvent molecules is introduced. We apply this description to the aqueous pair potential model. Our aim is limited to describe the important effects of the short-range interaction, and not to provide an accurate tool for their calculation. Therefore our model follows the PB theory as closely as possible, and describes the short-range interaction using a simplified term added to the free energy. The advantage of this approach over more elaborate treatments such as the AHNC 41, 42, is that it provides relatively simple equations that can be treated numerically and analytically with relative ease as well as allowing extensions to non-planar geometries. In a planar geometry, we show that the effect of the ion-ion hydration interaction can be understood as a perturbation over the PB results. An increase in the concentration of counter-ions near the charged surface is found, and it results in an apparent surface charge which is reduced relatively to the PB theory.

In addition to the calculation of charge distributions, the effect of the hydration interaction on the force between charged particles or surfaces can be studied and will be presented elsewhere [43]. Since we will not discuss inter-surface forces in this paper, it is worthwhile to mention that the results obtained using our model are in good agreement with AHNC calculations 44] and may provide an explanation to the hydration forces as observed in surface force measurements [31,32]. For high surface charge and plate separations up to approximately $20 \AA$, important modifications of the PB predictions are found.

The outline of the paper is as follows. Section [I] presents the model. In section III we apply the model to a single charged plate, present numerical results for the ion density profile and discuss the modifications to the PB theory due to the addition of short-range interactions. In section [V] we present analytical results in the low salt limit. We calculate the effective PB surface charge and the effect of the hydration interaction on the density profile of counter-ions in a system with no added salt. 


\section{THE MODEL}

\section{A. Free energy}

We start from an approximated free energy, written as a functional of the various ion densities. We choose the electrostatic boundary conditions to be of fixed surface charge densities and write the free energy as a sum of the usual PB term and a correction term, due to hydration, as will be explained below:

$$
\Omega=\Omega_{\mathrm{PB}}+\Delta \Omega
$$

We discuss first how the PB free energy is obtained, and then generalize this result to include the short-range hydration interaction.

\section{Poisson-Boltzmann free energy}

The Hamiltonian of the system is:

$$
H=\frac{1}{2} \sum_{i} \int_{V} \mathrm{~d}^{3} \mathbf{r} e_{i} \rho_{i}(\mathbf{r}) \phi(\mathbf{r})+\frac{1}{2} \oint_{\partial V} \mathrm{~d}^{2} \mathbf{r}_{\mathrm{s}} \sigma\left(\mathbf{r}_{\mathrm{s}}\right) \phi\left(\mathbf{r}_{\mathrm{s}}\right)
$$

where $V$ is the the volume occupied by the electrolyte solution, $\sigma\left(\mathbf{r}_{\mathrm{s}}\right)$ is the surface charge density of immobile charges on the boundaries $\partial V$, and $e_{i}$ is the charge of the $i$ th ion species. The ion densities $\rho_{i}(\mathbf{r})$ are:

$$
\rho_{i}(\mathbf{r}) \equiv \sum_{j=1}^{N_{i}} \delta\left(\mathbf{r}-\mathbf{r}_{i}^{j}\right)
$$

where $\mathbf{r}_{i}^{j}$ is the position of the $j$ th ion of the $i$ th species, and the electrostatic potential $\phi(\mathbf{r})$ is a function the different ion positions:

$$
\phi(\mathbf{r})=\sum_{j} \int_{V} \mathrm{~d}^{3} \mathbf{r}^{\prime} \frac{e_{j} \rho_{j}\left(\mathbf{r}^{\prime}\right)}{\varepsilon\left|\mathbf{r}-\mathbf{r}^{\prime}\right|}+\oint_{\partial V} \mathrm{~d}^{2} \mathbf{r}_{\mathrm{s}} \frac{\sigma\left(\mathbf{r}_{\mathrm{s}}\right)}{\varepsilon\left|\mathbf{r}-\mathbf{r}_{\mathrm{s}}\right|}
$$

where $\varepsilon=78$ is the dielectric constant of water. The PB theory is obtained by using a mean-field approximation for the electrostatic interaction. The Hamiltonian (2) is first replaced by a mean-field Hamiltonian, where the electrostatic potential $\phi(\mathbf{r})$ is replaced by an external field $\Psi(\mathbf{r})$. In the thermodynamic limit the free energy can then be written as a functional of the mean densities of the ion species, $c_{i}(\mathbf{r})=\left\langle\rho_{i}(\mathbf{r})\right\rangle_{\mathrm{MF}}$, as follows:

$$
\begin{aligned}
\Omega_{\mathrm{MF}}= & k_{B} T \int_{V} \sum_{i} c_{i}\left[\log \frac{c_{i}}{\zeta_{i}}-1\right] \mathrm{d}^{3} \mathbf{r} \\
& +\frac{1}{2} \int_{V} \sum_{i} e_{i} \Psi(\mathbf{r}) c_{i}(\mathbf{r}) \mathrm{d}^{3} \mathbf{r} \\
& +\frac{1}{2} \oint_{\partial V} \sigma\left(\mathbf{r}_{s}\right) \Psi\left(\mathbf{r}_{s}\right)
\end{aligned}
$$

where $k_{\mathrm{B}} T$ is the thermal energy, and $\zeta_{i}$ is the fugacity of the $i$ th ion species. The mean-field approximation is obtained by requiring that the external potential $\Psi(\mathbf{r})$ is the thermodynamical average of (4) in the system with the mean-field Hamiltonian, $\Psi(\mathbf{r})=\langle\phi(\mathbf{r})\rangle_{\mathrm{MF}}$, i.e.:

$$
\Psi(\mathbf{r})=\sum_{i} \int_{V} \mathrm{~d}^{3} \mathbf{r}^{\prime} \frac{e_{i} c_{i}\left(\mathbf{r}^{\prime}\right)}{\varepsilon\left|\mathbf{r}-\mathbf{r}^{\prime}\right|}+\oint_{\partial V} \mathrm{~d}^{2} \mathbf{r}_{\mathrm{s}} \frac{\sigma\left(\mathbf{r}_{\mathrm{s}}\right)}{\varepsilon\left|\mathbf{r}-\mathbf{r}_{\mathrm{s}}\right|}
$$

This relation is equivalent to the Poisson equation:

$$
\nabla^{2} \Psi=-\frac{4 \pi}{\varepsilon} \sum_{i} e_{i} c_{i}
$$

supplemented by the boundary condition:

$$
\left.\nabla \Psi \cdot \hat{\mathbf{n}}\right|_{\mathbf{r}_{\mathbf{s}}}=-\frac{4 \pi}{\varepsilon} \sigma\left(\mathbf{r}_{\mathbf{s}}\right) \quad \text { on the charged surfaces }
$$

where the normal vector $\hat{\mathbf{n}}$ points away from the charged surfaces into the volume occupied by the ionic solution. Using this boundary condition and Eq. (7), the second and third terms of Eq. (5) can be re-expressed as:

$$
\begin{aligned}
\frac{1}{2} \int_{V} \sum_{i} e_{i} \Psi(\mathbf{r}) c_{i}(\mathbf{r}) \mathrm{d}^{3} \mathbf{r} & +\frac{1}{2} \oint_{\partial V} \sigma\left(\mathbf{r}_{s}\right) \Psi(\mathbf{r}) \\
& =\frac{\varepsilon}{8 \pi} \int_{V}(\nabla \Psi)^{2} \mathrm{~d}^{3} \mathbf{r}
\end{aligned}
$$

Substituting this relation in Eq. (5) we obtain the PB free energy:

$$
\begin{aligned}
\Omega_{\mathrm{PB}} & =\frac{\varepsilon}{8 \pi} \int(\nabla \Psi)^{2} \mathrm{~d}^{3} \mathbf{r} \\
& +k_{B} T \int \sum_{i} c_{i}\left[\log \frac{c_{i}}{\zeta_{i}}-1\right] \mathrm{d}^{3} \mathbf{r} \\
& +\int \lambda(\mathbf{r})\left(\nabla^{2} \Psi+\frac{4 \pi}{\varepsilon} \sum_{i} e_{i} c_{i}\right) \mathrm{d}^{3} \mathbf{r}
\end{aligned}
$$

The first term in $\Omega_{\mathrm{PB}}$ is the electrostatic free energy and the second term is the entropy of the ions. The fugacity $\zeta_{i}$, in the second term, is equal in PB theory to the bulk concentration $c_{\mathrm{b}, i}$ of the $i$ th ion species, $\zeta_{i}=c_{\mathrm{b}, i}$, as for an ideal gas. For more generalized free energies, a different relation may exist between the fugacity of each ion species and its respective bulk concentration. The electrostatic potential $\Psi$ is a functional of the ion densities $c_{i}$, and is determined by the Poisson equation (7) and the boundary conditions (8) imposed by the surface charges. Alternatively, in Eq. (10) $\Psi$ is regarded as an independent field and a third term containing a Lagrange multiplier $\lambda(\mathbf{r})$ is added to $\Omega_{\mathrm{PB}}$. The $\mathrm{PB}$ equilibrium mean densities $c_{i}(\mathbf{r})$ result from minimizing $\Omega_{\mathrm{PB}}$. With the introduction of $\lambda(\mathbf{r})$ the minimization is equivalent to requiring an extremum of $\Omega_{\mathrm{PB}}$ with respect to the three fields $c_{i}, \Psi$ and $\lambda$, subject to the boundary condition ( 8 ). 
By requiring first an extremum of $\Omega_{\mathrm{PB}}$ with respect to $\Psi$ and $c_{i}$ the following relations are obtained:

$$
\lambda=\frac{\varepsilon}{4 \pi} \Psi
$$

and:

$$
c_{i}=\zeta_{i} \exp \left(-\beta e_{i} \Psi\right)
$$

Where $\beta=1 /\left(k_{\mathrm{B}} T\right)$ and $\zeta_{i}=c_{\mathrm{b}, i}$. The extremum condition with respect to $\lambda$ gives the Poisson equation:

$$
\nabla^{2} \Psi=-\frac{4 \pi}{\varepsilon} \sum_{i} e_{i} c_{i}
$$

Combining these relations we obtain the $\mathrm{PB}$ equation:

$$
\nabla^{2} \Psi=-\frac{4 \pi}{\varepsilon} \sum_{i} \zeta_{i} e_{i} \mathrm{e}^{-\beta e_{i} \Psi}
$$

Alternatively, the first two relations, obtained from the extremum condition with respect to $\Psi$ and $c_{\mathrm{i}}$, can be substituted into Eq. (10). Formally, this gives $\Omega_{\mathrm{PB}}$ as a functional of $\lambda$. Using Eq. (11), the expression obtained for $\Omega_{\mathrm{PB}}$ can be written as a functional of $\Psi$ :

$$
\begin{aligned}
\Omega_{\mathrm{PB}}= & -\frac{\varepsilon}{8 \pi} \int_{V}(\nabla \Psi)^{2} \mathrm{~d}^{3} \mathbf{r}+\oint_{\partial V} \sigma \Psi \mathrm{d}^{2} \mathbf{r}_{\mathrm{s}} \\
& -k_{\mathrm{B}} T \int_{V} \mathrm{~d}^{3} \mathbf{r} \sum_{i} \zeta_{i} \mathrm{e}^{-\beta e_{i} \Psi}
\end{aligned}
$$

where the second integration is over the charged surfaces. Requiring an extremum of this functional with respect to $\Psi$ is another way to obtain the Poisson-Boltzmann equation (14).

A more formal derivation of the mean field, PB free energy, and a discussion on its generalization to systems with non-electrostatic interactions is presented in Ref. 445]. The PB free energy (10) can also be derived by formulating the problem using field theory methods. In this approach the mean-field approximation is obtained as the saddle point of the functional integral, and corrections due to ion-ion correlations can be obtained in a systematic expansion [19,20].

\section{Inclusion of the hydration interaction}

As discussed in the introduction, our starting point is a model in which the hydration interaction, arising from solvent effects, is described as an effective ion-pair interaction. We denote this short-range potential between ions of species $i$ and $j$ at distance $\mathbf{r}$ as $u_{i j}(\mathbf{r})$. The potential is taken as the short-range effective potential between ions immersed in a bulk ionic solution having a specific, constant concentration. Therefore, $u_{i j}(\mathbf{r})$ is assumed to be isotropic and does not depend on the ion positions or the confining geometry.
Our aim is to treat the long range electrostatic interaction on the mean-field level, as in PB theory. Thus, we begin by considering the free energy of a system placed in some arbitrary field $\Psi(\mathbf{r})$, where the ions interact with each other only through the two-body potential $u_{i j}(\mathbf{r})$. Due to the short-range nature of the hydration interaction, the free energy can be obtained from a virial expansion of the grand canonical partition function. Since we will be interested in highly inhomogeneous systems, we perform an expansion in the inhomogeneous ion density. The derivation is given in Appendix A. Including terms up to the quadratic order in the expansion we obtain:

$$
\begin{aligned}
\Omega_{\mathrm{h}}= & k_{\mathrm{B}} T \int \sum_{i} c_{i}\left[\log \frac{c_{i}}{\zeta_{i}}-1\right] \mathrm{d}^{3} \mathbf{r} \\
& +\int \sum_{i} e_{i} c_{i} \Psi \mathrm{d}^{3} \mathbf{r} \\
& +\frac{k_{B} T}{2} \sum_{i, j} \int c_{i}(\mathbf{r}) U_{i j}\left(\mathbf{r}-\mathbf{r}^{\prime}\right) c_{j}\left(\mathbf{r}^{\prime}\right) \mathrm{d}^{3} \mathbf{r d}^{3} \mathbf{r}^{\prime}
\end{aligned}
$$

where $\Psi(\mathbf{r})$ is an external field, coupled to the $i$ th ion charge density $e_{i} c_{i}$. The short-range weighted potential $U_{i j}$ in the third term of $\Omega_{\mathrm{h}}$ is defined as:

$$
U_{i j}=1-\mathrm{e}^{-\beta u_{i j}\left(\left|\mathbf{r}-\mathbf{r}^{\prime}\right|\right)}
$$

where $u_{i j}$ is the nominal short-range interaction potential between ions of species $i$ and $j$. This form of describing the short-range interaction is a rather crude approximation, valid only in the low density limit. Its advantage is its simplicity. The free energy $\Omega_{\mathrm{h}}$ amounts to setting the direct correlation function $c_{2}\left(\left|\mathbf{r}-\mathbf{r}^{\prime}\right|\right)$ to be equal to $-U\left(\left|\mathbf{r}-\mathbf{r}^{\prime}\right|\right)$, and all higher order direct correlation functions to zero 46$]$.

Having found the hydration free energy $\Omega_{\mathrm{h}}$, the electrostatic interaction can be treated on the mean-field level. This is done by considering $\Psi(\mathbf{r})$ as the electrostatic potential and imposing the self-consistency requirement of the Poisson equation (7). This is essentially the approximation we used to derive the PB equation (14), with the difference that the free energy of a dilute, noninteracting ion distribution is replaced by the free energy $\Omega_{\mathrm{h}}$ of Eq. (16). The result is the free energy of Eq. (1), with $\Delta \Omega$ defined as follows:

$$
\Delta \Omega=\frac{k_{B} T}{2} \sum_{i, j} \int c_{i}(\mathbf{r}) U_{i j}\left(\mathbf{r}-\mathbf{r}^{\prime}\right) c_{j}\left(\mathbf{r}^{\prime}\right) \mathrm{d}^{3} \mathbf{r} \mathrm{d}^{3} \mathbf{r}^{\prime}
$$

We conclude this section with some remarks on the approach presented above. Important solvent effects are already introduced in the PB theory by using an electrostatic interaction with a dielectric constant $\varepsilon=78$ of water, instead of the bare electrostatic interaction. In the modified model a more precise effective potential between the ions is used. The separation of this potential into a long-range electrostatic term and a short-range hydration term allows each of these two interactions to 
be treated in a simple though approximated form. The virial expansion is a standard choice for approximating short-range interactions. Such an expansion fails for the electrostatic interaction due to its long-range [47. On the other hand, the wide success of PB theory demonstrates that the electrostatic interaction can be treated quite well in the mean-field approximation. Therefore we use this approximation for the long-range part of the interaction, and in this respect we remain within the framework of PB theory.

The free energy (11) can also be obtained by rewriting the grand canonical partition function as a field-theory partition function. The short-range part of the interaction can be separated from the electrostatic interaction and a different expansion can be performed for each of these interactions. By using a density expansion for the short-range interaction and a loop expansion for the electrostatic interaction, Eq. (11) is obtained up to second order in the density expansion and first order in the electrostatic potential [48].

The simplicity of our approach can lead to elegant analytical results, but has several limitations. The use of only the second term in the virial expansion implies that we are using a low density approximation. The validity of such an approximation for a bulk fluid can be assessed by considering $k_{\mathrm{B}} T B_{2} c$, where $B_{2}$ is the second virial coefficient in the expansion of the pressure, and $c$ is the ion density. Qualitatively, if $B_{2} c$ is small compared to unity, the correction to the ideal gas behavior is small and truncating the virial expansion after the second term is sensible. For non-homogeneous cases, the corresponding quantity is $(1 / 2) \sum_{j} \int \mathrm{d} \mathbf{r}^{\prime} c\left(\mathbf{r}^{\prime}\right) U_{i j}\left(\mathbf{r}-\mathbf{r}^{\prime}\right)$. For relatively high surface charges considered here this integral approaches values of order unity near the charged surfaces, indicating that the approximation should only be expected to give qualitative results. Another deficiency of the virial expansion to second order can be seen from the fact that the direct correlation function is simply $-U_{i j}(\mathbf{r})$. This implies that the hard core interaction is not described accurately in our treatment. A faithful description would require the vanishing of the pair correlation function $h_{2}(\mathbf{r})$ for separations smaller than the hard-core diameter. Hence, it should be kept in mind that our main concern is to study the effects of a short-range interaction with a dominant attractive part. Finally, the fact that we describe the electrostatic interaction in the mean-field approximation implies that ion-ion correlations are ignored, as they are in PB theory. When our approach is applied for the aqueous pair potential model, these approximations should also be kept in mind. In particular, we follow Ref. 34 and do not include an effective ion-surface potential [49].

\section{B. Density equations}

The mean density distribution is obtained by minimizing the total free energy $\Omega=\Omega_{\mathrm{PB}}+\Delta \Omega$. From equations (11), (10) and (18) we have:

$$
\begin{aligned}
\Omega= & \frac{\varepsilon}{8 \pi} \int(\nabla \Psi)^{2} \mathrm{~d}^{3} \mathbf{r}+k_{B} T \int \sum_{i} c_{i}\left(\log \frac{c_{i}}{\zeta_{i}}-1\right) \mathrm{d}^{3} \mathbf{r} \\
& +\frac{k_{B} T}{2} \sum_{i, j} \int c_{i}(\mathbf{r}) U_{i j}\left(\mathbf{r}-\mathbf{r}^{\prime}\right) c_{j}\left(\mathbf{r}^{\prime}\right) \mathrm{d}^{3} \mathbf{r d}^{3} \mathbf{r}^{\prime} \\
& +\int \lambda(\mathbf{r})\left(\nabla^{2} \Psi+\frac{4 \pi}{\varepsilon} \sum_{i} c_{i} e_{i}\right) \mathrm{d}^{3} \mathbf{r}
\end{aligned}
$$

where $\mu_{i}$ and $\zeta_{i}=\exp \left(\beta \mu_{i}\right) / \lambda_{\mathrm{T}}^{3}$ are the chemical potential and the fugacity of the ion species $i$, respectively. The thermal de Broglie wavelength, $\lambda_{\mathrm{T}}$, is equal to $h /\left(2 \pi m k_{\mathrm{B}} T\right)^{1 / 2}$, where $h$ is the Planck constant and $m$ is the ion mass. Requiring an extremum of $\Omega$ with respect to $\Psi$ gives: $\lambda=(\varepsilon / 4 \pi) \Psi$ as in Eq. (11). Taking the variation with respect to $c_{i}$ then gives:

$$
\log \frac{c_{i}(\mathbf{r})}{\zeta_{i}}+\sum_{j} \int c_{j}\left(\mathbf{r}^{\prime}\right) U_{i j}\left(\mathbf{r}-\mathbf{r}^{\prime}\right) \mathrm{d}^{3} \mathbf{r}^{\prime}+\beta e_{i} \Psi(\mathbf{r})=0
$$

This equation is supplemented by the Poisson equation (7). Since Eq. (20) is an integral equation, the $c_{i}$ cannot be written as a simple function of $\Psi$ as in the PB case. Therefore, a single equation for $\Psi$, analogous to the PB equation, cannot be obtained, and we are left with the two coupled integro-differential equations (20) and (7). These equations should be solved together to obtain the electrostatic potential and density profiles. In the case $U \rightarrow 0$, Eq. (20) reduces to the Boltzmann relation $c_{i}=\zeta_{i} \exp \left(-\beta e_{i} \Psi\right)$ with $\zeta_{i}=c_{\mathrm{b}, i}$. Combining this relation with Eq. (7) reproduces the PB equation (14).

In order to simplify the set of equations, we assume the same short-range interaction between the different pairs of ion species. Assuming that the charged surfaces are negatively charged, we choose: $u_{i j}(\mathbf{r})=u_{++}(\mathbf{r}) \equiv u(\mathbf{r})$, where $u_{++}(\mathbf{r})$ is the short-range effective potential between the (positive) counter-ions. This assumption is not exact for the effective potentials of ions in water [39]. However, since only the counter-ions reach high densities, close to the oppositely charged surfaces, and the co-ions are repelled from the surface neighborhood, the exact choice of the potentials $u_{+-}(\mathbf{r})$ and $u_{--}(\mathbf{r})$ is expected to be of only minor significance.

We now consider an electrolyte of valency $z_{+}: z_{-}$, i.e., a solution of positive and negative ions of charges $e_{ \pm}=$ $\pm z_{ \pm} e$, where $e$ is the electron charge. We designate the surface charge density on the plate as a constant $\sigma$ and the bulk densities of the positive and negative ions as $c_{\mathrm{b}} \equiv c_{\mathrm{b},+}$ and $c_{\mathrm{b},-}$, respectively. Due to charge neutrality in the bulk, $c_{\mathrm{b},-}=\left(z_{+} / z_{-}\right) c_{\mathrm{b}}$ and similarly, 
$\zeta_{-}=\left(z_{+} / z_{-}\right) \zeta$ where $\zeta \equiv \zeta_{+}$. Equation (20) can then be written as follows:

$$
c_{ \pm}(\mathbf{r})=\zeta_{ \pm} e^{\mp \beta e z_{ \pm} \Psi} \exp \left[-\int c\left(\mathbf{r}^{\prime}\right) U\left(\mathbf{r}-\mathbf{r}^{\prime}\right) \mathrm{d}^{2} \mathbf{r}^{\prime}\right]
$$

where $c(\mathbf{r})=c_{+}(\mathbf{r})+c_{-}(\mathbf{r})$ is the total ion density, and $U(\mathbf{r})=U_{++}(\mathbf{r})$ is obtained from $u(\mathbf{r})$ using Eq. (17). From the Poisson equation (7) we obtain:

$$
\begin{aligned}
\nabla^{2} \Psi= & -\frac{4 \pi e}{\varepsilon}\left(z_{+} c_{+}-z_{-} c_{-}\right) \\
= & \frac{4 \pi e}{\varepsilon} \zeta z_{+}\left(\mathrm{e}^{\beta e z_{-} \Psi}-\mathrm{e}^{-\beta e z_{+} \Psi}\right) \\
& \quad \times \exp \left[-\int c\left(\mathbf{r}^{\prime}\right) U\left(\mathbf{r}-\mathbf{r}^{\prime}\right) \mathrm{d}^{3} \mathbf{r}^{\prime}\right]
\end{aligned}
$$

Note that in addition to the explicit dependence on the ion valencies $z_{ \pm}$in equations (21) and (22), in a more realistic model the details of the potential $u(\mathbf{r})$ should also depend on the type of counter-ion species present in the problem.

\section{SINGLE CHARGED PLATE}

\section{A. Density equations}

After presenting the general formalism let us consider, as an example, a single negatively charged planar surface (Fig. 1). The charged surface is in contact with an electrolyte of valency $z_{+}: z_{-}$. We designate the axis perpendicular to the plate as the $z$ axis, and consider the ion solution in the region $z>0$. For simplicity we consider positive and negative ions of the same hard-core diameter $d_{\mathrm{hc}}$. The coordinate of closest approach of the ions to the plate is designated as $z=0$. Hence the "real" surface lies at a distance of one ion radius $d_{\mathrm{hc}} / 2$ from the actual $z=0$ plate position, as shown in Fig. 1a. When we refer to conventional PB results, however, the ions are pointlike and the plate should be understood to be positioned exactly at $z=0$.

Due to the one-dimensional symmetry imposed by the uniformly charged planar plate, the integration in Eq. (21) can be performed over the $x-y$ plane, leaving us with profiles depending only on $z$, the distance from the plate:

$$
c_{ \pm}(z)=\zeta_{ \pm} e^{\mp \beta e z_{ \pm} \Psi} \exp \left[-\int_{0}^{\infty} c\left(z^{\prime}\right) B\left(z-z^{\prime}\right) \mathrm{d} z^{\prime}\right]
$$

where $c(z)=c_{+}(z)+c_{-}(z)$ is the total ion density and $B(z)$ is the integral of $U(\mathbf{r})$ in the plane of constant $z$. Using cylindrical coordinates:

$$
B(z)=2 \pi \int_{0}^{\infty} \rho \mathrm{d} \rho U\left(\sqrt{z^{2}+\rho^{2}}\right)
$$

and the Poisson equation (22) reads:

$$
\begin{aligned}
& \frac{\mathrm{d}^{2} \Psi}{\mathrm{d} z^{2}}=\frac{4 \pi e}{\varepsilon} \zeta z_{+}\left(\mathrm{e}^{\beta e z_{-} \Psi}-\mathrm{e}^{-\beta e z_{+} \Psi}\right) \\
& \quad \times \exp \left[-\int_{0}^{\infty} c\left(z^{\prime}\right) B\left(z-z^{\prime}\right) \mathrm{d} z^{\prime}\right]
\end{aligned}
$$

Equations (23) and (25) are supplemented by the boundary conditions:

$$
\left.\frac{\mathrm{d} \Psi}{\mathrm{d} z}\right|_{z=0}=-\frac{4 \pi}{\varepsilon} \sigma ;\left.\quad \frac{\mathrm{d} \Psi}{\mathrm{d} z}\right|_{z \rightarrow \infty}=0
$$

Finally, the relation between $\zeta$ and the bulk density $c_{\mathrm{b}}$ can be obtained from Eq. (23). As $z \rightarrow \infty, \Psi$ becomes zero, and $c_{ \pm}$assume their asymptotic constant, bulk values. Thus the integrand inside the exponential can be replaced by $-\left(1+z_{+} / z_{-}\right) c_{\mathrm{b}} B\left(z-z^{\prime}\right)$. Recalling that $c_{+}=c_{\mathrm{b}}$ and $c_{-}=\left(z_{+} / z_{-}\right) c_{\mathrm{b}}$, we obtain:

$$
c_{\mathrm{b}}=\zeta \exp \left[-\left(1+\frac{z_{+}}{z_{-}}\right) B_{\mathrm{t}} c_{\mathrm{b}}\right]
$$

where:

$$
B_{\mathrm{t}} \equiv \int_{-\infty}^{\infty} \mathrm{d} z B(z)=\int \mathrm{d}^{3} \mathbf{r} U(\mathbf{r})
$$

is also equal to $2 B_{2}$, the second virial coefficient. Note that $B(z)$ and $B_{\mathrm{t}}$ are negative for an attractive interaction. The limit $B_{\mathrm{t}} c_{\mathrm{b}} \rightarrow 0$ is the limit in which the short-range interaction becomes negligible in the bulk. In this limit the relation between the bulk density and fugacity of Eq. (27) tends to the ideal gas relation $c_{\mathrm{b}}=$ $\zeta=\exp (\beta \mu) / \lambda_{\mathrm{T}}^{3}$.

Two special cases will be of particular interest in the following sections. The first is the case of a monovalent 1:1 electrolyte, where we have:

$$
\begin{aligned}
& c_{ \pm}(z)=\zeta \mathrm{e}^{\mp \beta e \Psi} \exp \left[-\int_{0}^{\infty} c\left(z^{\prime}\right) B\left(z-z^{\prime}\right) \mathrm{d} z^{\prime}\right] \\
& \frac{\mathrm{d}^{2} \Psi}{\mathrm{d} z^{2}}=-\frac{4 \pi}{\varepsilon} c(z)
\end{aligned}
$$

and:

$$
c_{\mathrm{b}}=\zeta \exp \left(-2 B_{\mathrm{t}} c_{\mathrm{b}}\right)
$$

The second case is that of no added salt. The solution contains only monovalent counter-ions $\left(z_{+}=1, z_{-}=0\right)$. This case can be obtained by taking formally the limit $\zeta \rightarrow 0$ of Eq. (29), or by repeating the derivation from Eq. (19) with only one type of ions, of charge $e$. The term $-k_{\mathrm{B}} T \int \mathrm{d}^{3} \mathrm{r} c \log (\zeta)$ in $\Omega$ is then a Lagrange multiplier added to impose the condition: $\int_{0}^{\infty} \mathrm{d} z \operatorname{ec}(z)=|\sigma|$. The following equations are then obtained:

$$
\begin{aligned}
c(z) & =\zeta_{0} \mathrm{e}^{-\beta e \Psi} \exp \left[-\int_{0}^{\infty} c\left(z^{\prime}\right) B\left(z-z^{\prime}\right) \mathrm{d} z^{\prime}\right] \\
\frac{\mathrm{d}^{2} \Psi}{\mathrm{d} z^{2}} & =-\frac{4 \pi e}{\varepsilon} c(z)
\end{aligned}
$$


where $\zeta_{0}$ is an arbitrary reference fugacity. The choice of $\zeta_{0}$ determines the (arbitrary) position in which $\Psi$ is zero. Note that the electrostatic potential $\Psi$ diverges in the bulk. This divergence exists also in the usual PB theory, because the system is effectively one dimensional with no screening by added salt. Although $\Psi(z)$ has a weak logarithmic divergence, the density of counter-ions decays to zero, $\lim _{z \rightarrow \infty} c(z)=0$ as it should.

\section{B. Parameters and length scales}

For the ion-ion potential $u\left(\mathbf{r}-\mathbf{r}^{\prime}\right)$ we use an effective potential between $\mathrm{Na}^{+}-\mathrm{Na}^{+}$ion pairs. The potential was calculated using a Monte-Carlo simulation [39] for an $\mathrm{NaCl}$ ionic solution of concentration $0.55 \mathrm{M}$, at room temperature. The electrostatic interaction between the ions is subtracted, and the net short-range potential is shown in Fig. 2. For ion-ion separations below $2.9 \AA$ a hard core interaction is assumed. Fig. 3 shows the function $B(z)$, derived from this potential, using Eq. (24). Note that $B(z)$ has several local maxima and minima. These correspond to the local maxima and minima of $u(\mathbf{r})$. Thus the structure of $B(z)$ reflects the oscillatory behavior of the effective potential.

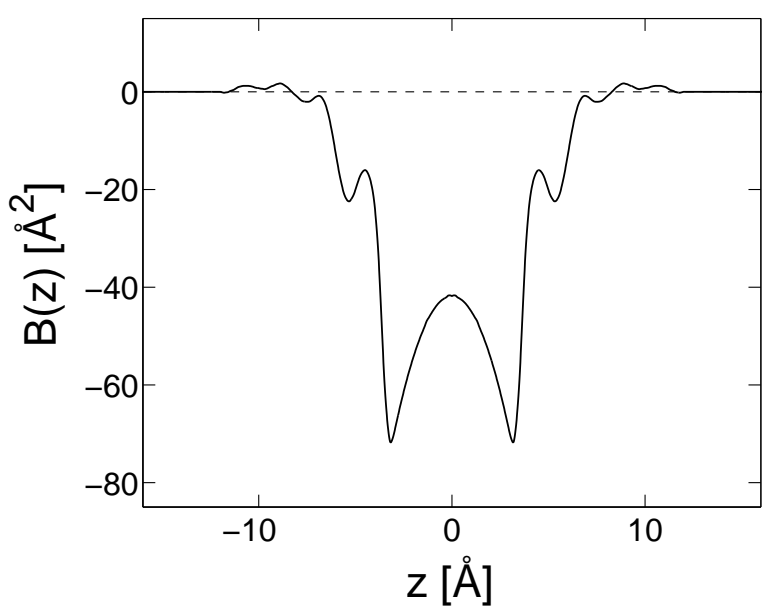

FIG. 3. The effective interaction in a planar geometry $B(z)$ obtained from the potential of Fig. 2, using Eq. (24). The oscillating structure of the radial potential shown in Fig. 2 is apparent in the secondary minima of $B(z)$.

We use the effective potential calculated for $c_{\mathrm{b}}=$ $0.55 \mathrm{M}$, regardless of the actual bulk ion concentration in the system. Since the important effects occur near the charged surface, where the ion concentration is much larger than $c_{\mathrm{b}}$, it seems reasonable to use an effective potential calculated in the presence of a rather high salt concentration. The choice of $c_{\mathrm{b}}=0.55 \mathrm{M}$ is still somewhat arbitrary, and we rely on the fact that the dependence of the effective potential on the ion concentration is weak 39].

It is useful to employ two length scales that charac- terize the PB density profiles [6]. The Gouy-Chapman length, defined as $b=\varepsilon k_{\mathrm{B}} T /(2 \pi e|\sigma|)$, characterizes the width of the diffusive counter-ion layer close to a single plate charged with a surface charge $\sigma$, in the absence of added salt. The Debye-Hückel screening length, $\lambda_{\mathrm{D}}=$ $\left(8 \pi c_{\mathrm{b}} e^{2} / \varepsilon k_{\mathrm{B}} T\right)^{-1 / 2}$, equal to $19.6 \AA$ for $c_{\mathrm{b}}=0.025 \mathrm{M}$ at room temperature characterizes the decay of the screened electrostatic interaction in a solution with added salt. The strength of the electrostatic interaction can also be expressed using the Bjerrum length, $l_{\mathrm{B}}=e^{2} /\left(\varepsilon k_{\mathrm{B}} T\right)$. This is the distance at which the electrostatic interaction between two unit charges becomes equal to the thermal energy. The Bjerrum length is equal to about $7 \AA$ in water at room temperature.

The inclusion of hydration interactions introduces additional length scales in the system. For the interaction shown in Figs. 2 and 3, the range of the interaction $d_{\text {hyd }}$ can be seen to be approximately $7 \AA$, over twice the hard core diameter $d_{\mathrm{hc}}=2.9 \AA$. The strength of the hydration interaction is characterized by $B_{\mathrm{t}} \simeq-(7.9 \AA)^{3}$, as is calculated from Eq. (28).

\section{Numerical results}

Equations (23) and (25) are a set of three nonlinear integro-differential equations. We treat them numerically using an iterative scheme, based on the assumption that the positive ion density profile is dominated by the electrostatic interaction. We start with the analytically known PB profile close to a single charged plate and calculate iteratively corrections to this profile, as result from equations (23) and (25). For a 1:1 electrolyte we iteratively solve the equation:

$$
\begin{aligned}
\frac{\mathrm{d}^{2} \Psi^{(n)}}{\mathrm{d} z^{2}}= & \frac{8 \pi e}{\varepsilon} \zeta \sinh \left(\beta e \Psi^{(n)}\right) \\
& \times \exp \left[-\int_{0}^{\infty} c^{(n-1)}\left(z^{\prime}\right) B\left(z-z^{\prime}\right) \mathrm{d} z^{\prime}\right]
\end{aligned}
$$

where $c(z)=c_{+}(z)+c_{-}(z)$ is the total ion density and the superscript $n$ stands for the $n$th iteration. For $n>0$ :

$$
\begin{aligned}
c_{ \pm}^{(n)}(z) \equiv & \zeta \mathrm{e}^{\mp \beta e \Psi^{(n)}} \\
& \times \exp \left[-\int_{0}^{\infty} c^{(n-1)}\left(z^{\prime}\right) B\left(z-z^{\prime}\right) \mathrm{d} z^{\prime}\right]
\end{aligned}
$$

and the zeroth order densities $c_{ \pm}^{(0)}$ are taken as the density profiles generated by the $\mathrm{PB}$ equation (14). The boundary conditions $(26)$ are satisfied by the electrostatic potential $\Psi^{(n)}$ in all the iterations. Note that using our iterative scheme, Eq. (32) is an inhomogeneous differential equation, because the integral in the exponential is a known function of $z$, calculated numerically in the $(n-1)$ iteration. A similar iterative scheme, based on Eq. 31 can be used when only counter-ions are present in the solution. 


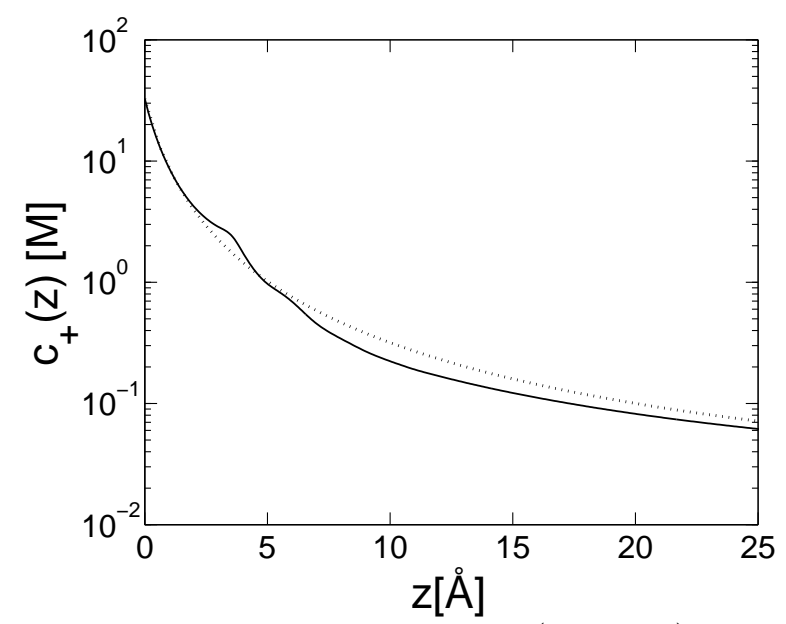

FIG. 4. Counter-ion density profile (solid line) obtained from numerical solution of Eq. (31) with the hydration interaction as of Fig. 3, plotted on a semi-log plot. No salt is present in the solution. The surface charge is $|\sigma|=0.333 \mathrm{C} / \mathrm{m}^{2}$. The dielectric constant is $\varepsilon=78$ and the temperature is $T=298 \mathrm{~K}$. The dotted line shows the corresponding density profile obtained from the PB equation.

Figure 4 shows the calculated density profile of the counter-ions on a semi-logarithmic scale, for a charged plate with a surface charge, $|\sigma|=0.333 \mathrm{C} / \mathrm{m}^{2}$, corresponding to an area of approximately $48 \AA^{2}$ per unit charge. This is a typical high surface charge obtained with mica plates. It corresponds to a Gouy-Chapman length $b=1.06 \AA$, at a temperature of $298 \mathrm{~K}$, with $\varepsilon=78$. No salt is present in the solution. The calculated density profile (solid line) is compared to the PB prediction (dotted line). The short-range attraction favors an increased concentration of counter-ions in the vicinity of the charged plate. This results in an increase of the concentration relative to the $\mathrm{PB}$ prediction. For a surface charge as in Fig. 4, an increase of the concentration is seen at distances from the plate up to approximately $4.5 \AA$. The overall number of counter-ions is fixed by the requirement of charge neutrality. Therefore, the increase in the density of counter-ions near the plate is balanced by a reduced concentration further away.

When salt is present in the solution, the short-range attraction draws additional ions from the bulk solution to the diffuse electrical layer near the plate. This can be seen in Fig. 5, in a comparison of counter-ion profiles for different values of the bulk concentration $c_{\mathrm{b}}$. For each salt concentration, the figure shows the ratio between the counter-ion density and the density predicted by PB theory, as a function of the distance from the plate. The dotted line shows the result in the no-salt limit. As the salt concentration increases, the counterion concentration increases relative to the $\mathrm{PB}$ concentration at all distances from the charged plate. Qualitatively, however, the hydration effect on the counter-ion profile is similar in all the curves. As long as the Debye-Hückel screening length is large compared to the Gouy-Chapman length,

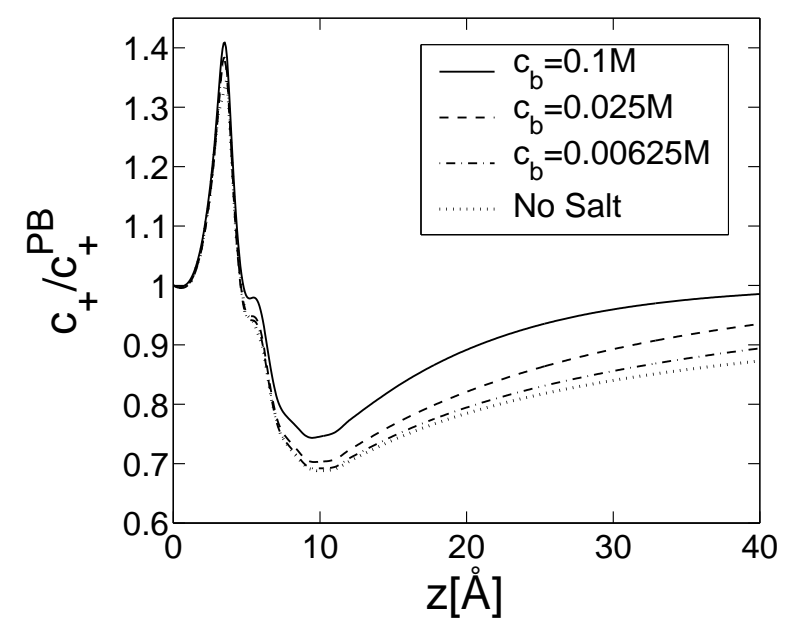

FIG. 5. The ratio $c_{+} / c_{+}^{\mathrm{PB}}$ between the positive ion density obtained from Eq. (29) and the value obtained from PB theory, for a surface charge $|\sigma|=0.333 \mathrm{C} / \mathrm{m}^{2}$ and several values of $c_{\mathrm{b}}$. Other parameters are as in Fig. 4. The three values of $c_{\mathrm{b}}: 0.1 \mathrm{M}, 0.025 \mathrm{M}$ and $0.00625 \mathrm{M}$ correspond to Debye-Hückel screening lengths $\lambda_{\mathrm{D}} \simeq 9.8 \AA, 19.6 \AA$ and $39.2 \AA$, respectively.

$b=1.06 \AA$, the density profile in the vicinity of the plate is dominated by the balancing counter-ions and the salt has only a small effect.

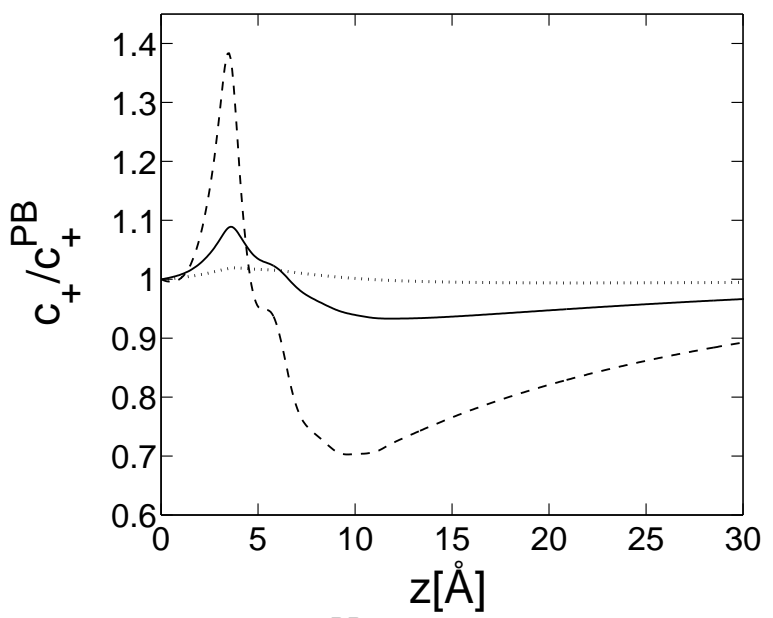

FIG. 6. The ratio $c_{+} / c_{+}^{\mathrm{PB}}$ between the positive ion density obtained from Eq. (29) and the value obtained from PB theory, for surface charges $|\sigma|=0.333 \mathrm{C} / \mathrm{m}^{2}$ (dashed line), $0.1 \mathrm{C} / \mathrm{m}^{2}$ (solid line) and $0.0333 \mathrm{C} / \mathrm{m}^{2}$ (dotted line). The bulk salt concentration $c_{\mathrm{b}}$ is $0.025 \mathrm{M}$. Other parameters are as in Fig. 4.

The effect of the hydration interaction is strongly dependent on the surface charge $\sigma$. As $\sigma$ is increased, the ion density near the surface increases too. The exponential in Eq. (31) deviates more strongly from unity, leading to a larger deviation from PB theory. The dependence on $\sigma$ is demonstrated in Fig. 6. The ratio of the positive ion density to its PB value is shown for three values of the surface charge. The effect of the hydration potential 
is very minor for small surface charge, $|\sigma|=0.0333 \mathrm{C} / \mathrm{m}^{2}$ (dotted line), where the deviation from PB is less than $2 \%$ at its maximum, and considerable for a surface charge of $0.333 \mathrm{C} / \mathrm{m}^{2}$ (dashed line), where the deviation from PB reaches almost $40 \%$.

The numerical scheme, described above, requires several iterations to converge fully. It is interesting to note, though, that the first iteration captures most of the effect of the short range interaction. This indicates that the density profile is dominated, as we assumed, by the electrostatic interaction, and assures that the convergence of the iterative scheme is good with the PB density profile as the zero-th order approximation. On the theoretical level it indicates that the effect of the hydration interaction can be seen as a perturbation over the PB results. The fact that the first iteration provides a good approximation to the full iterative result can lead to further analytical approximations. For example, the corrections to the density profiles, in the no added salt limit, are studied analytically in the next section, based on this observation.

As an example for the results of the first iteration, we compare, in Fig. 7, the correction to the counter-ion density profile obtained in the first iteration (dashed line), with the full iterative result (solid line). We use a high surface charge of $0.333 \mathrm{C} / \mathrm{m}^{2}$, where the differences between the exact profile and that of the first iteration are relatively pronounced. The two density profiles differ by at most 3.2 percent, where the ion density deviates from the $\mathrm{PB}$ value by 30 percent. For smaller surface charge the results obtained in the first iteration are even better.

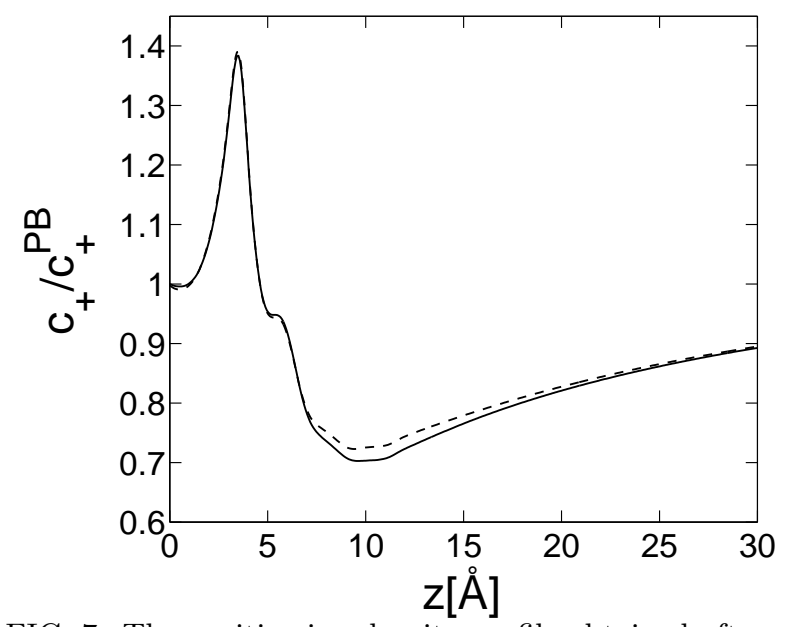

FIG. 7. The positive ion density profile obtained after one iteration of Eq. (32) (dotted line), compared to the full solution of Eq. (29) (solid line). Parameters are as in Fig. 5. The maximal deviation between the two density profiles is 3.2 percent, where the deviation from $\mathrm{PB}$ is approximately 30 percent.

\section{Contact density and the contact theorem}

The contact density of the ions is barely modified as compared with the $\mathrm{PB}$ prediction. This is evident in Figs. 4-6. As long as the Debye-Hückel screening length is large compared to the Gouy-Chapman length, or the hydration interaction is negligible in the bulk, the modification remains small. This result can be obtained from a generalization of the $\mathrm{PB}$ contact theorem [5,50]:

$$
\sum_{i} c_{i}(0)-\frac{2 \pi \beta}{\varepsilon} \sigma^{2}=P_{\text {bulk }}
$$

where $P_{\text {bulk }}$ is the bulk pressure of the ionic solution. Equation (34) is derived in detail for the free energy used in our model in Ref. 43. It is obtained from the equality of the internal pressure in the electrolyte solution at different distances from the charged plate. Far away from the charged plate the pressure must be equal to the bulk pressure of the ionic solution, because the densities approach their bulk values and the electrostatic potential becomes constant. At the contact plane between the plate and the solution, the pressure involves only an electrostatic contribution and an osmotic contribution, as in PB theory. This is due to the fact that in our model no short range interaction between the plate and the ions is included. Equating the pressure at the contact plane and far away from the plate results in Eq. (34).

The contact density, as expressed by Eq. (34), differs from the PB prediction only due to the change in the actual value of $P_{\text {bulk }}$. This change is negligible if the shortrange interaction is not of importance in the bulk. In addition, if the surface charge is high, such that $b \ll \lambda_{\mathrm{D}}$, $P_{\text {bulk }}$ is negligible compared to the second term in the left hand side of Eq. (34). Thus the contact density remains very close to the $\mathrm{PB}$ prediction. In the no-salt limit $P_{\text {bulk }}$ is zero and the contact density coincides exactly with the $\mathrm{PB}$ result, $c_{+}(0)=(2 \pi \beta / \varepsilon) \sigma^{2}$.

\section{ANALYTICAL SOLUTIONS}

The simplicity of the model makes it possible to obtain various analytical results. The effect of the hydration on the ion distribution can be characterized by several quantities, such as the magnitude of the deviation from the PB result and the effective $\mathrm{PB}$ surface charge density seen at a distance from the plate. Using several simplifying assumptions it is possible to obtain analytical expressions for these quantities.

First we assume that the hydration interactions can be neglected in the bulk, i.e., $B_{\mathrm{t}} c_{\mathrm{b}} \ll 1$. In this case, the effect of the hydration potential is significant only in the vicinity of the charged surface, where the ion density becomes large. In addition, the Debye-Hückel screening length, $\lambda_{\mathrm{D}}$, is taken to be large compared to the GouyChapman length $b=e /\left(2 \pi l_{\mathrm{B}}|\sigma|\right)$. Since $\lambda_{\mathrm{D}} \gg b$, the 
negative co-ion density near the negatively charged surface can be neglected compared to the positive counterion density. Far away from the charged plate, the system is well described using the PB equation, with an effective surface charge density $\sigma_{\text {eff }}$ different from the actual charge density $\sigma$. The result of the above two simplifying assumptions is that the salt is of minor importance in the region where the effective surface charge is determined. The effective surface charge can then be inferred by considering the case in which only counter-ions are present in the solution (no added salt).

Equation (31) can now be recast in a simpler form, by considering $\eta \equiv \log \left(c / \zeta_{0}\right)$, as expressed by Eq. (31), and taking its second derivative:

$$
\frac{\mathrm{d}^{2} \eta}{\mathrm{d} z^{2}}=\frac{4 \pi}{\varepsilon} \beta e^{2} \zeta_{0} \mathrm{e}^{\eta}-\int_{0}^{\infty} \zeta_{0} \mathrm{e}^{\eta\left(z^{\prime}\right)} \frac{\mathrm{d}^{2} B\left(z-z^{\prime}\right)}{\mathrm{d} z^{2}} \mathrm{~d} z^{\prime}
$$

The PB density profile, $c_{\mathrm{PB}}(z) \equiv \zeta_{0} \mathrm{e}^{\eta_{0}(z)}$, for the same surface charge, satisfies the equation $d^{2} \eta_{0} / d z^{2}=$ $\left(4 \pi \beta e^{2} \zeta_{0} / \varepsilon\right) \exp \left(\eta_{0}\right)$. Its exact solution is known to be:

$$
c_{\mathrm{PB}}(z)=\zeta_{0} \mathrm{e}^{\eta_{0}(z)}=\frac{1}{2 \pi l_{\mathrm{B}}} \cdot \frac{1}{(z+b)^{2}}
$$

Note that only in the $\mathrm{PB}$ equation $\eta(z)$ is the reduced electrostatic potential $e \Psi(z) / k_{\mathrm{B}} T$. From the generalized contact theorem (34), the surface density in the no added salt case and in the presence of one plate is $c(0)=2 \pi \beta \sigma^{2} / \varepsilon$, as in PB theory. Therefore:

$$
\eta(z=0)=\eta_{0}(z=0)
$$

From the derivative of $c(z)$, Eq. (31), we find:

$$
\frac{\mathrm{d} \eta}{\mathrm{d} z}=-\beta e \frac{\mathrm{d} \Psi}{\mathrm{d} z}-\int_{0}^{\infty} \mathrm{d} z^{\prime} c\left(z^{\prime}\right) \frac{\mathrm{d} B\left(z-z^{\prime}\right)}{\mathrm{d} z}
$$

and using the boundary condition (26):

$$
\left.\frac{\mathrm{d} \eta}{\mathrm{d} z}\right|_{z=0}=\left.\frac{\mathrm{d} \eta_{0}}{\mathrm{~d} z}\right|_{z=0}+\int_{0}^{\infty} \mathrm{d} z^{\prime} c\left(z^{\prime}\right) \frac{\mathrm{d} B\left(z^{\prime}\right)}{\mathrm{d} z}
$$

where the odd parity of $\mathrm{d} B / \mathrm{d} z$ has been used. This relation can be used together with Eq. (37) as a second boundary condition at $z=0$, instead of the boundary condition of vanishing $\mathrm{d} \eta / \mathrm{d} z$ at infinity.

Linearizing Eq. (35) with respect to:

$$
w \equiv \eta-\eta_{0}=\log \left(c / c_{\mathrm{PB}}\right)
$$

which is valid for relatively small deviations from the PB profile, results in the following equation:

$$
\begin{aligned}
& \frac{\mathrm{d}^{2} w}{\mathrm{~d} z^{2}}-\frac{4 \pi}{\varepsilon} \beta e^{2} c_{\mathrm{PB}}(z) w(z) \\
& =-\int_{0}^{\infty} \mathrm{d} z^{\prime}\left(1+w\left(z^{\prime}\right)\right) c_{\mathrm{PB}}\left(z^{\prime}\right) \frac{\mathrm{d}^{2} B\left(z-z^{\prime}\right)}{\mathrm{d} z^{2}}
\end{aligned}
$$

This equation can be further simplified by omitting $w\left(z^{\prime}\right)$ from the integrand in the right hand side. This approximation was motivated in Sec. III C and is equivalent to stopping the iterative scheme (32) after the first iteration. The density profile is then replaced by the PB density profile in the term that involves the hydration interaction $B(z)$. This results in the equation:

$$
\frac{\mathrm{d}^{2} w}{\mathrm{~d} z^{2}}-\frac{4 \pi}{\varepsilon} \beta e^{2} c_{\mathrm{PB}}(z) w(z)+\Gamma(z)=0
$$

where $\Gamma(z)$ is the convolution integral:

$$
\Gamma(z)=\frac{1}{2 \pi l_{\mathrm{B}}} \int_{0}^{\infty} \mathrm{d} z^{\prime} \frac{1}{\left(z^{\prime}+b\right)^{2}} \frac{\mathrm{d}^{2} B\left(z-z^{\prime}\right)}{\mathrm{d} z^{2}}
$$

The corresponding boundary conditions, obtained from equations (37) and (39) using the same approximations, are:

$$
\begin{aligned}
& w(z=0)=0 \\
& \left.\frac{\mathrm{d} w}{\mathrm{~d} z}\right|_{z=0}=\int_{0}^{\infty} \mathrm{d} z^{\prime} c_{\mathrm{PB}}\left(z^{\prime}\right) \frac{\mathrm{d} B\left(z^{\prime}\right)}{\mathrm{d} z}
\end{aligned}
$$

Equation (42) is a second order linear differential equation for $w(z)$ and can be solved analytically. The solution, given in detail in Appendix B, is expressed in terms of the convolution integral $\Gamma(z)$ of Eq. 43 . The effective surface charge and the effect of the hydration on the density profile can then be calculated in several limits, described in detail in Appendix B. Here we outline the main results.

\section{A. Slowly varying density: $b \gg d_{\mathrm{hyd}}$}

In the limit $b \gg d_{\text {hyd }}$, the $\mathrm{PB}$ distribution varies slowly on the scale of the hydration interaction, described by $B(z)$, and the theory becomes effectively a local density functional theory. The specific form of $B(z)$ is not important, and all the results simply depend on $B_{\mathrm{t}}=\int_{-\infty}^{\infty} B(z) \mathrm{d} z$. The deviation of the effective Gouy-Chapman length $b_{\text {eff }}$ from the actual GouyChapman length $b$ depends linearly on $B_{t}$ and on the surface charge $\sigma \sim 1 / b$. This can be expected since we use a linearized equation. Thus we have, on dimensional grounds, $b_{\text {eff }}-b \sim B_{t} / l_{B} b$. The detailed calculation gives the numerical prefactor:

$$
b_{\mathrm{eff}}-b \cong \frac{-B_{\mathrm{t}}}{4 \pi l_{\mathrm{B}}} \frac{1}{b}
$$

Since $B_{\mathrm{t}}$ is negative $b_{\text {eff }}$ is larger than $b$ and the effective surface charge, $\sigma_{\text {eff }}$, is smaller than the actual surface charge $\sigma$. This result should be expected. The short range interaction attracts counterions to the vicinity of the charged plate and the surface charge is screened more effectively than in the PB equation.

The correction to the counter-ion density profile, described by $w(z)=\log \left[c(z) / c_{\mathrm{PB}}(z)\right]$, is found to be: 


$$
w(z)=\frac{-B_{\mathrm{t}}}{2 \pi l_{\mathrm{B}}}\left\{\frac{3}{2(z+b)^{2}}-\frac{1}{b(z+b)}\right\}
$$

The density profile is increased relative to $\mathrm{PB}$ theory for distances smaller than $b / 2$, and decreased for larger distances. The deviation from $\mathrm{PB}, w(z)$, is maximal at $z=0$, where it is equal to $-B_{\mathrm{t}} /\left(4 \pi l_{\mathrm{B}} b^{2}\right)$, and minimal at $z=2 b$, where it is equal to $B_{\mathrm{t}} /\left(12 \pi l_{\mathrm{B}} b^{2}\right)$.

Figure 8 shows the approximated function $w(z)$ of Eq. (46) for $b=21.2 \AA$, corresponding to $b / d_{\text {hyd }} \approx 3$ (dotted line). The approximation is compared with the function $w(z)$ obtained from the exact solution of equation (31) for the case of no added salt (solid line). Although $b$ is not much larger than $d_{\text {hyd }}$, the approximation describes well the correction to the $\mathrm{PB}$ profile. Note that $w(z)$, as expressed by Eq. (46) is maximal at $z=0$, whereas according to the contact theorem $w(0)$ should be zero. This apparent inconsistency results from neglecting the range of the hydration potential relative to $b$. In the precise solution of Eq. (31) $w(0)$ is zero, as it should be. The prediction of Eq. (46) is valid only for distances $z \gtrsim d_{\mathrm{hyd}}$, as can be seen in Figure 8 .

The range of validity of the linearization procedure can be found by requiring that the minimal and maximal values of $w(z)$ are small compared to unity:

$$
\frac{-B_{\mathrm{t}}}{4 \pi l_{\mathrm{B}} b^{2}} \ll 1
$$

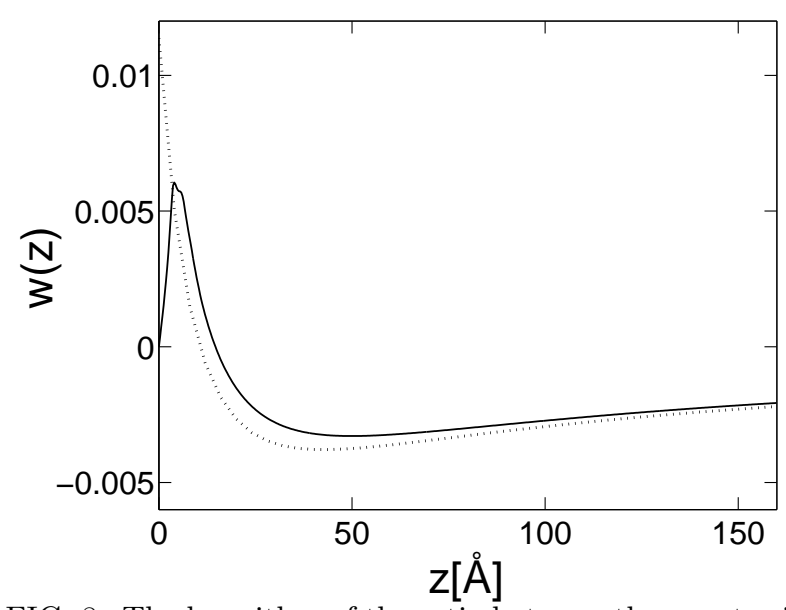

FIG. 8. The logarithm of the ratio between the counter-ion density obtained with the inclusion of the hydration interaction and its value in PB theory, $w(z)$, as a function of the distance from a charged plate, with no added salt in the solution. The solid line shows the function $w(z)$ obtained from the exact solution, for $b=21.2 \AA$. The dotted line shows the approximated curve obtained from the linearization with respect to $w$, Eq. (42), in the limit $b \gg d_{\text {hyd }}$, Eq. (46).

\section{B. Surface layer limit: $b \ll d_{\text {hyd }}$}

In the limit in which $b \ll d_{\text {hyd }}$, the ion density effectively becomes a dense layer concentrated at $z=0$ on the scale of the hydration interaction. The effective GouyChapman length has the same form as in the limit of slowly varying density, $b \gg d_{\text {hyd }}$, but having a different prefactor:

$$
b_{\mathrm{eff}}-b \cong \frac{-B_{\mathrm{t}}}{12 \pi l_{\mathrm{B}}} \frac{1}{b}
$$

The effective surface charge is, therefore, smaller than the actual surface charge. Note that $b_{\text {eff }}$ depends on $B(z)$, in this limit, only through $B_{\mathrm{t}}$. The linear dependence on $\sigma \sim 1 / b$ follows from the linearization leading to Eq. (42), as in the previous limit.

It should be stressed that although $b$ is small compared to $d_{\text {hyd }}$ we still assume that $b$ is large enough for the linearization to be valid, i.e., we assume that $w(z)$ is small compared to unity. Furthermore, the counter-ion density should be small enough that we can sensibly use only the quadratic term in the virial expansion. To check the validity of these assumptions, the correction to the density profile should be considered.

The form of $w(z)$ depends, in the surface layer limit, on the specific form of $B(z)$. In order to study $w(z)$ analytically, we use an approximated form of $B(z)$, described in Appendix B. A typical form of the approximated $w(z)$, obtained using this approximation [Eq. (B12)], is shown in Fig. 9 (dotted line). The Gouy-Chapman length is $b=1.06 \AA$, corresponding to $b / d_{\text {hyd }} \approx 0.15$. In addition, the function $w(z)$ obtained from the exact solution of equation (31) is shown for comparison (solid line). The approximated curve captures well the qualitative behavior of the correction to the PB profile. Note that the discrepancy between the approximated and actual profiles results not only from the linearization and small $b$ limit, but also from the loss of detail due to the use of an approximated form for $B(z)$.

The deviation from the PB profile, $w(z)$, can be qualitatively described as follows. For $z<d_{\mathrm{hc}}, w(z)$ increases from zero quadratically (with an additional term of the form $z^{2} \log z$ ) to its value at $z=d_{\mathrm{hc}}$. It then decreases from its maximum positive value to a minimum, negative value, on a scale of the range of the attractive part of $B(z)$. This minimum value is equal to approximately $B_{\mathrm{t}} / 6 \pi l_{\mathrm{B}} b d_{\mathrm{hyd}}$. For distances larger than the interaction range, $w(z)$ assumes the form $w(z) \sim 1 / z$, characterizing a PB profile with a modified, effective surface charge. For finite values of $b$, we can expect the above behavior to be smoothed over a scale of order $b$. 


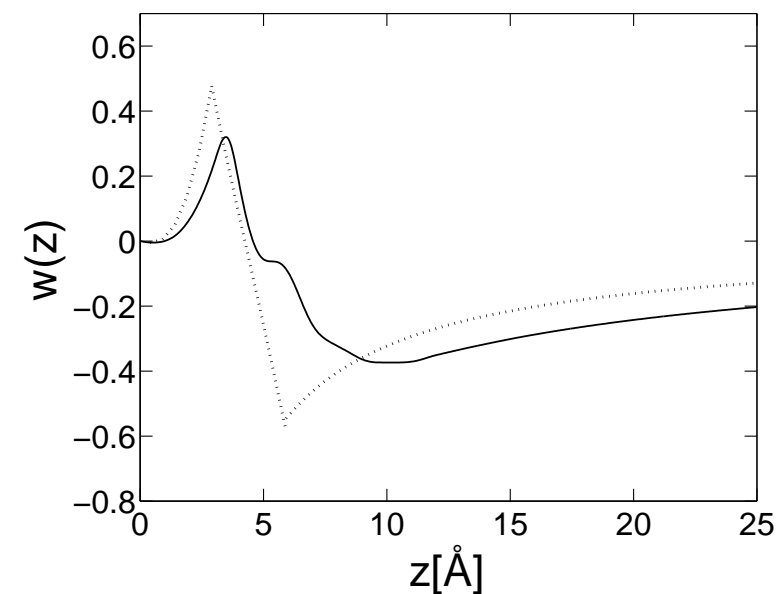

FIG. 9. The logarithm of the ratio between the counter-ion density obtained with the inclusion of the hydration interaction and its value in PB theory, $w(z)$, as a function of the distance from a charged plate, with no added salt in the solution. The solid line shows the function $w(z)$ obtained from the exact solution, for $b=1.06 \AA$. The dotted line shows the approximated curve obtained from the linearization with respect to $w$, Eq. (42), in the limit $b \ll d_{\text {hyd }}$, Eq. (B12).

The validity of the linearization can be found by requiring that $|w(z)| \ll 1$. This requirement results in the following condition:

$$
\frac{-B_{\mathrm{t}}}{6 \pi l_{\mathrm{B}} b d_{\mathrm{hyd}}} \ll 1
$$

The validity of stopping the virial expansion at the quadratic order can be shown to have the same condition. For the hydration potential of Fig. 2, the condition expressed in Eq. (49) implies that the various approximations we use start to break down when $b$ becomes smaller than approximately $1 \AA$, or $\sigma \gtrsim 0.022 e / \AA^{2}$. When $b$ is of this order, it is well below $d_{\text {hyd }}$, making the surface layer limit a sensible approximation.

\section{Effective surface charge}

In the two limits described above, the effective GouyChapman length was found to be of the form $b_{\text {eff }}-b \sim$ $-B_{t} / l_{\mathrm{B}} b$, with different prefactors in the two limits. For intermediate values of $b$, the effective charge depends on the specific structure of the function $B(z)$. In order to study this dependence, we use a simple approximated form for $B(z)$, described in Appendix B. Using this approximation, an analytical expression can be obtained for the effective Gouy-Chapman length for all values of $b$.

Figures 10a and 10b show the predicted $b_{\text {eff }}$ and $b_{\text {eff }}-b$, respectively (solid lines) as a function of $b$, together with the asymptotic limits (45) and (48) (dotted lines). As the surface charge increases from zero (and $b$ decreases from infinity), the effective charge $\left|\sigma_{\text {eff }}\right|$ increases too (but is always smaller than the actual surface charge). When $b$ reaches a certain value $b^{\text {min }}, b_{\text {eff }}$ starts increasing with further reduction of $b$, i.e., the effective charge decreases with increasing surface charge above $|\sigma|^{\max }=$ $e /\left(2 \pi l_{\mathrm{B}} b^{\mathrm{min}}\right)$. The value of $b^{\mathrm{min}}$ depends on the structure of the function $B(z)$, but can be estimated to be between the values predicted by the asymptotic expressions (45) and (48). From the condition $\mathrm{d} b_{\mathrm{eff}} /\left.\mathrm{d} b\right|_{b=b^{\min }}=0$ we find:

$$
\sqrt{\frac{-B_{\mathrm{t}}}{12 \pi l_{\mathrm{B}}}}<b^{\min }<\sqrt{\frac{-B_{\mathrm{t}}}{4 \pi l_{\mathrm{B}}}}
$$

and:

$$
b_{\mathrm{eff}}^{\min } \simeq 2 b^{\min }
$$

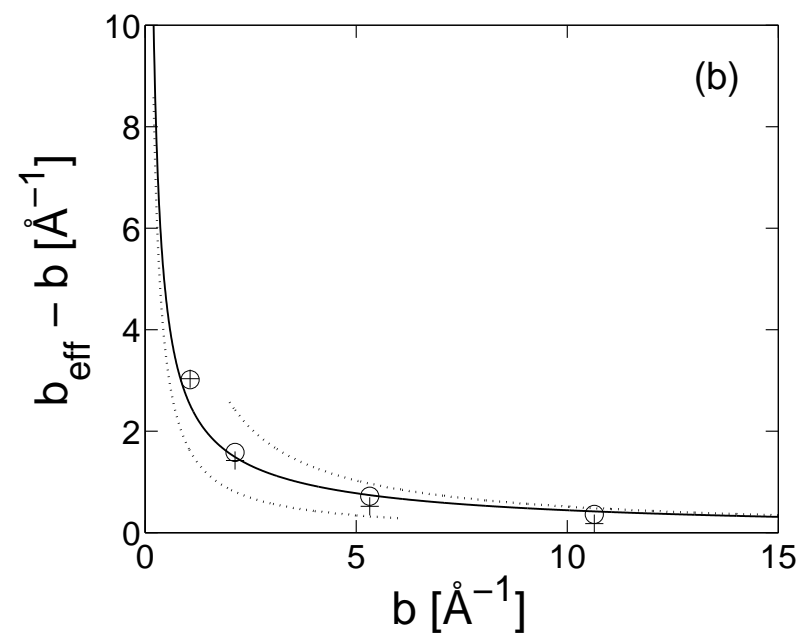

FIG. 10. The effective Gouy-Chapman length $b_{\text {eff }}(\mathrm{a})$ and $\Delta b=b_{\text {eff }}-b(\mathrm{~b})$, as a function of the Gouy-Chapman length $b$. The solid lines show the behavior predicted by Eq. (B14), with $B_{\mathrm{t}}=-500 \AA^{3}, d_{\mathrm{hc}}=2.9 \AA$ and $B_{0}=41.8 \AA^{2}$. The dotted lines show the asymptotic limits of equations (45) and (48). The symbols show results extracted from numerical solutions of Eq. (29), using $B(z)$ of Fig. 3, with salt concentrations of $10^{-7} \mathrm{M}$ (circles) and $0.1 \mathrm{M}$ (crosses). The salt has a very small effect. 
For the hydration interaction of Fig. 2, $B_{\mathrm{t}}$ is approximately $-500 \AA^{3}$. The value of $b^{\text {min }}$ is then between $1.36 \AA$ and $2.35 \AA$, corresponding to a surface charge density between $0.15 \mathrm{C} / \mathrm{m}^{2}$ and $0.26 \mathrm{C} / \mathrm{m}^{2}$. The values obtained from the approximated curve, shown in Fig. 10, are $b^{\text {min }} \simeq 1.5 \AA$ and $b_{\text {eff }}^{\min } \simeq 3.4 \AA$.

For small enough values of $b$, the effective surface charge $\left|\sigma_{\text {eff }}\right|$ should increase again with an increase of $|\sigma|$ and become larger than $|\sigma|$. This effect cannot be predicted by our model because of the low density approximation used for the hard core interaction. In particular, the hard core of the ions should cause the density to saturate at the close packing density, leading to a reduced screening of the surface charge relative to $\mathrm{PB}$ theory 25,26,51. In our model, as in the PB theory, the counterion density near the surface is not bounded, and increases indefinitely as $\sigma$ is increased. Although our model includes the steric repulsion between ions, this repulsion is "softened", and is always outweighed by the attractive part of the ion-ion interaction.

In addition to the prediction obtained using the linearized approximation, Figure 10 shows values of $b_{\text {eff }}$ extracted from numerical solutions of the full equation (29), using the original interaction $B(z)$. The equation was solved with two different salt concentrations: $10^{-7} \mathrm{M}$ (circles) and $0.1 \mathrm{M}$ (crosses). The value of $b_{\text {eff }}$ was estimated from the positive ion density at large distances from the plate, by finding the value of $b$ that would result in the same calculated values of the density in a solution of the PB equation. Note that for both salt concentrations, $b_{\text {eff }}$ is very close to its predicted value, meaning that the salt has a very small effect on $\sigma_{\text {eff }}$. This result is not obvious for the high salt concentration of $0.1 \mathrm{M}$. The Debye-Hückel screening length is approximately $9.6 \AA$, not much larger than the range of the hydration interaction, $d_{\text {hyd }} \simeq 7 \AA$, and comparable to the Gouy-Chapman length at the large $b$ region of the plot.

\section{CONCLUSIONS AND OUTLOOK}

In this work we have studied the effects due to the discreteness of the solvent in aqueous ionic solutions. Hydration interactions are found to have a significant effect on the structure of the diffusive layer near highly charged surfaces. The counter-ion density is increased in the vicinity of the charged surface, relative to the PB prediction, and decreased further away. The distance from the charged plate in which the density is increased, and the magnitude of the deviation from the PB density, depend strongly on the surface charge, and on the parameters of the short-range hydration interaction between ion pairs.

The ion-ion hydration interaction can be described roughly using two parameters. The first parameter is the range of the hydration interaction, $d_{\text {hyd }}$, equal to approximately $7 \AA$ for $\mathrm{Na}^{+}-\mathrm{Na}^{+}$pairs. The second parameter, $B_{\mathrm{t}}$ has dimensions of volume and characterizes the strength of the hydration interaction. It is equal to approximately $-500 \AA^{3}$ for $\mathrm{Na}^{+}-\mathrm{Na}^{+}$pairs. Two limits can be considered, where the Gouy-Chapman length, $b \sim 1 / \sigma$, is small or large compared to the range of the hydration interaction $d_{\text {hyd }}$. In both of these limits we assume that the Debye-Hückel screening length, $\lambda_{\mathrm{D}}$, is large compared to $b$ and $d_{\text {hyd }}$.

In the limit $b \gg d_{\text {hyd }}$, the counter-ion density becomes depleted, relative to the $\mathrm{PB}$ prediction, starting at a distance $z \simeq b / 2$ from the charged plate. The maximum absolute value of $w(z)=\log \left[c(z) / c_{\mathrm{PB}}(z)\right]$ scales as $-B_{\mathrm{t}} / l_{\mathrm{B}} b^{2}$. In the limit $b \ll d_{\text {hyd }}$, the distance from the plate, where the counter-ion density becomes lower than the PB prediction, is between $z=d_{\text {hc }}$ and $z=d_{\text {hyd }}$. The maximum absolute value of $w(z)$ scales as $-B_{\mathrm{t}} / l_{\mathrm{B}} d_{\text {hyd }} b$.

Far away from the charged plate, the density profile can be well described using the PB theory with an effective surface charge that can be calculated analytically. The correction to the Gouy-Chapman length in the two limits $b \gg d_{\text {hyd }}$ and $b \ll d_{\text {hyd }}$ is always positive and scales as $-B_{\mathrm{t}} / l_{\mathrm{B}} b$, but has different numerical prefactors. When the surface charge on the plate is increased, the effective surface charge, $\sigma_{\text {eff }}$, is found to reach a certain maximal value. Above this maximal value $\sigma_{\text {eff }}$ decreases with further increase of the actual $\sigma$ on the plate. The various approximations we use start to break down when $b$ is smaller than approximately $-B_{\mathrm{t}} / 6 \pi l_{\mathrm{B}} d_{\mathrm{hyd}}$, corresponding to $b \lesssim 1 \AA$.

An important outcome of this work is that the correction of the PB ion density due to the hydration interaction is significant near highly charged surfaces. The electrostatic interaction dominates the ionic distribution and the hydration interaction can be seen as a perturbation. For a high surface charge density of, say, one unit charge per $48 \AA^{2}$ the counterion density deviates from its Poisson Boltzmann value by at most 30 percent. The effective change in the surface charge is more significant, from $1 e / 48 \AA^{2}$ to about $1 e / 13 \AA^{2}$.

The hydration effect on inter-surface forces can be very pronounced, as opposed to the effect on the ion distribution. This result will be presented elsewhere [43]. Our model predicts an attractive contribution to the pressure between two parallel charged plates. At distances below several nanometers this contribution can outweigh the electrostatic repulsion and lead to an overall attraction between the plates. Our two-plate findings can also be compared with available AHNC results 41, 44, showing good qualitative agreement both for the ion density profile and pressure.

The formalism we present can be readily generalized to other geometries. This could lead to an estimation of the aqueous solvent effects on phenomena such as the Manning condensation on cylindrical polyions [52], and charge renormalization of spherical mycelles or colloids [9]. In this respect our formalism offers an advantage over the AHNC approximation which was applied so far 
only in a planar geometry. Another interesting extension of this work would be to consider the combination of fluctuation and hydration effects. This is particularly important for ionic solutions with divalent counter-ions, where fluctuation effects become large 21,22,24.

\section{ACKNOWLEDGMENTS}

We wish to thank S. Marčelja for introducing us to the subject of solvent effects in aqueous ionic solutions, and for valuable discussions and suggestions. We would like to thank R. Netz, H. Orland and R. Podgornik for useful discussions. Partial support from the U.S.-Israel Binational Foundation (B.S.F.) under grant No. 98-00429, and the Israel Science Foundation founded by the Israel Academy of Sciences and Humanities - Centers of Excellence Program is gratefully acknowledged.

\section{APPENDIX A: INHOMOGENEOUS VIRIAL EXPANSION}

We consider an inhomogeneous system of particles with a short-range two-body interaction, and aim to express the free energy of the system in the low density limit as a functional of the density distribution. For simplicity we consider only one species of particles. The inhomogeneity of the system arises from the inclusion of an external field $\varphi(\mathbf{r})$, or from the boundary conditions imposed on the system. We begin by considering the grand canonical ensemble. The grand canonical partition function is:

$$
Z_{\mathrm{G}}=\sum_{N} \frac{1}{N !}\left(\frac{\mathrm{e}^{\beta \mu}}{\lambda_{\mathrm{T}}^{3}}\right)^{N} Q_{N}
$$

where $\mu$ is the chemical potential, $\lambda_{\mathrm{T}}$ is the de Broglie thermal wavelength and $Q_{N}$ is:

$$
\begin{gathered}
Q_{N}=\int \prod_{i=1}^{N} \mathrm{~d}^{3} \mathbf{r}_{i} \mathrm{e}^{-\beta U_{N}\left(\left\{\mathbf{r}_{i}\right\}\right)} \\
U_{N}\left(\left\{\mathbf{r}_{i}\right\}\right)=\sum_{i} \varphi\left(\mathbf{r}_{i}\right)+\frac{1}{2} \sum_{i} \sum_{j \neq i} u\left(\left|\mathbf{r}_{i}-\mathbf{r}_{j}\right|\right)
\end{gathered}
$$

We proceed on similar lines as the usual virial expansion in a bulk fluid, expanding $\log Z_{\mathrm{G}}$ in powers of the activity. Up to second order we have:

$$
\begin{aligned}
\log Z_{\mathrm{G}}= & \left(\frac{\mathrm{e}^{\beta \mu}}{\lambda_{\mathrm{T}}^{3}}\right) Q_{1}+\frac{1}{2}\left(\frac{\mathrm{e}^{\beta \mu}}{\lambda_{\mathrm{T}}^{3}}\right)^{2}\left(Q_{2}-Q_{1}^{2}\right)=\left(\frac{\mathrm{e}^{\beta \mu}}{\lambda_{\mathrm{T}}^{3}}\right) \int \mathrm{d}^{3} \mathbf{r} \mathrm{e}^{-\beta \varphi(\mathbf{r})} \\
& +\frac{1}{2}\left(\frac{\mathrm{e}^{\beta \mu}}{\lambda_{\mathrm{T}}^{3}}\right)^{2} \int \mathrm{d}^{3} \mathbf{r} \int \mathrm{d}^{3} \mathbf{r}^{\prime} \mathrm{e}^{-\beta\left(\varphi(\mathbf{r})+\varphi\left(\mathbf{r}^{\prime}\right)\right)}\left(\mathrm{e}^{-\beta u\left(\left|\mathbf{r}-\mathbf{r}^{\prime}\right|\right)}-1\right)
\end{aligned}
$$

This can be seen as an expansion in powers of the field $\exp [\beta(\mu-\varphi(\mathbf{r}))] / \lambda_{\mathrm{T}}^{3}$. The local density $c(\mathbf{r})$ can be expressed in a similar expansion:

$$
\begin{aligned}
c(\mathbf{r})= & -\frac{1}{\beta} \frac{\delta \log Z_{\mathrm{G}}}{\delta \varphi(\mathbf{r})}=\left(\frac{\mathrm{e}^{\beta \mu}}{\lambda_{\mathrm{T}}^{3}}\right) \mathrm{e}^{-\beta \varphi(\mathbf{r})} \\
& +\left(\frac{\mathrm{e}^{\beta \mu}}{\lambda_{\mathrm{T}}^{3}}\right)^{2} \mathrm{e}^{-\beta \varphi(\mathbf{r})} \int \mathrm{d}^{3} \mathbf{r}^{\prime} \mathrm{e}^{-\beta \varphi\left(\mathbf{r}^{\prime}\right)}\left(\mathrm{e}^{-\beta u\left(\left|\mathbf{r}-\mathbf{r}^{\prime}\right|\right)}-1\right)
\end{aligned}
$$

This relation can be inverted to obtain an expansion of $\exp [\beta(\mu-\varphi(\mathbf{r}))] / \lambda_{\mathrm{T}}^{3}$ in powers of $c(\mathbf{r})$. Up to the second order:

$$
\frac{\mathrm{e}^{\beta(\mu-\varphi(\mathbf{r}))}}{\lambda_{\mathrm{T}}^{3}}=c(\mathbf{r})+c(\mathbf{r}) \int \mathrm{d}^{3} \mathbf{r}^{\prime} c\left(\mathbf{r}^{\prime}\right)\left(1-\mathrm{e}^{-\beta u\left(\left|\mathbf{r}-\mathbf{r}^{\prime}\right|\right)}\right)
$$


and by substituting this relation in Eq. (A4) $\log Z_{\mathrm{G}}$ can be expressed as an expansion in $c$. Up to the second order:

$$
\log Z_{\mathrm{G}}=\int \mathrm{d}^{3} \mathbf{r} c(\mathbf{r})+\frac{1}{2} \int \mathrm{d}^{3} \mathbf{r} \int \mathrm{d}^{3} \mathbf{r}^{\prime} c(\mathbf{r}) c\left(\mathbf{r}^{\prime}\right)\left(1-\mathrm{e}^{-\beta u\left(\left|\mathbf{r}-\mathbf{r}^{\prime}\right|\right)}\right)
$$

The grand canonical potential can be obtained from the relation $\Omega=-k_{\mathrm{B}} T \log Z_{\mathrm{G}}$, with $\log Z_{\mathrm{G}}$ given by Eq. (A7). In this expression, $c(\mathbf{r})$ is the mean density profile for the imposed external field $\varphi(\mathbf{r})$ and a given chemical potential $\mu$. We would like to express $\Omega$ as a functional of a general ion density $c(\mathbf{r})$, whose minimization with respect to $c(\mathbf{r})$ would give the equilibrium mean density. Regarding $-k_{\mathrm{B}} T \log Z_{\mathrm{G}}$ as a functional of $\chi(\mathbf{r}) \equiv \varphi(\mathbf{r})-\mu$, we have:

$$
-k_{\mathrm{B}} T \frac{\delta \log Z_{\mathrm{G}}}{\delta \chi(\mathbf{r})}=c(\mathbf{r})
$$

The Legendre transform of this relation can be obtained by defining:

$$
\Theta=-k_{\mathrm{B}} T \log Z_{\mathrm{G}}-\int \mathrm{d}^{3} \mathbf{r} c(\mathbf{r}) \chi(\mathbf{r})
$$

and expressing $\log Z_{\mathrm{G}}$ and $\chi$ as functionals of $c(\mathbf{r})$. We have already expressed $\log Z_{\mathrm{G}}$ as a functional of $c(\mathbf{r})$ in Eq. (A7). An expression for $\chi(\mathbf{r})$ as a functional of $c(\mathbf{r})$ can be obtained from Eq. (A6). Up to first order in $c$ we have :

$$
\begin{aligned}
\beta[\varphi(\mathbf{r})-\mu] & =-\log \left\{\lambda_{\mathrm{T}}^{3} c(\mathbf{r})\left[1+\int \mathrm{d}^{3} \mathbf{r}^{\prime} c\left(\mathbf{r}^{\prime}\right)\left(1-\mathrm{e}^{-\beta u\left(\left|\mathbf{r}-\mathbf{r}^{\prime}\right|\right)}\right)\right]\right\} \\
& =-\log \left[\lambda_{\mathrm{T}}^{3} c(\mathbf{r})\right]-\int \mathrm{d}^{3} \mathbf{r}^{\prime} c\left(\mathbf{r}^{\prime}\right)\left(1-\mathrm{e}^{-\beta u\left(\left|\mathbf{r}-\mathbf{r}^{\prime}\right|\right)}\right)+\mathrm{O}\left(c^{2}\right)
\end{aligned}
$$

Using this relation and Eq. A7 we obtain, up to second order in c:

$$
\begin{aligned}
\beta \Theta(\{c(\mathbf{r})\})= & \int \mathrm{d}^{3} \mathbf{r} c(\mathbf{r})\left[\log \left(\lambda_{\mathrm{T}}^{3} c(\mathbf{r})\right)-1\right] \\
& +\frac{1}{2} \int \mathrm{d}^{3} \mathbf{r} \int \mathrm{d}^{3} \mathbf{r}^{\prime} c(\mathbf{r}) c\left(\mathbf{r}^{\prime}\right)\left(1-\mathrm{e}^{-\beta u\left(\left|\mathbf{r}-\mathbf{r}^{\prime}\right|\right)}\right)
\end{aligned}
$$

The functional $\Theta$ of $c(\mathbf{r})$ has the property that:

$$
\frac{\delta \Theta}{\delta c(\mathbf{r})}=-\chi(\mathbf{r})=-[\varphi(\mathbf{r})-\mu]
$$

or equivalently:

$$
\frac{\delta}{\delta c(\mathbf{r})}\left\{\Theta+\int \mathrm{d}^{3} \mathbf{r} c(\mathbf{r})[\varphi(\mathbf{r})-\mu]\right\}=\frac{\delta \Omega(\{c(\mathbf{r})\})}{\delta c(\mathbf{r})}=0
$$

Thus, using Eq. (A11), we obtain:

$$
\begin{aligned}
\Omega(\{c(\mathbf{r})\})= & k_{\mathrm{B}} T \int \mathrm{d}^{3} \mathbf{r} c(\mathbf{r})\left(\log \frac{c(\mathbf{r})}{\zeta}-1\right)+\int \mathrm{d}^{3} \mathbf{r} c(\mathbf{r}) \varphi(\mathbf{r}) \\
& +\frac{1}{2} k_{\mathrm{B}} T \int \mathrm{d}^{3} \mathbf{r} \int \mathrm{d}^{3} \mathbf{r}^{\prime} c(\mathbf{r}) c\left(\mathbf{r}^{\prime}\right)\left(1-\mathrm{e}^{-\beta u\left(\left|\mathbf{r}-\mathbf{r}^{\prime}\right|\right)}\right)
\end{aligned}
$$

where $\zeta=\exp (\beta \mu) / \lambda_{\mathrm{T}}^{3}$.

The derivation of Eq. (A14) can be readily generalized to the case of several ion species of different charges and different pair interactions $u_{i j}(\mathbf{r})$, resulting in Eq. (16).

A similar, more elaborate diagrammatic expansion of the thermodynamic potentials in the presence of an external field is presented in Ref. [53]. A variational principal for the grand canonical potential $\Omega$ is obtained in which $\Omega$ is expressed as a functional of the mean density $c(\mathbf{r})$ and the pair correlation function $h_{2}\left(\mathbf{r}_{1}, \mathbf{r}_{2}\right)$. This expression is equivalent to Eq. (A14) up to the second order in the cluster expansion. 


\section{APPENDIX B: DETAILS OF ANALYTICAL RESULTS}

In this appendix we present details of the analytical approximations of Sec. IV.

We consider first the analytical solution of Equation (42). This equation is a second order linear differential equation for $w(z)$. Note that the function $c_{\mathrm{PB}}(z)$ is a known function of $z$, given by Eq. (36). The solution of Eq. (42), with the boundary conditions of Eq. (44) is:

$$
w(z)=\frac{1}{z+b} \int_{0}^{z} \mathrm{~d} z_{2}\left(z_{2}+b\right)^{2} \int_{z_{2}}^{\infty} \frac{\mathrm{d} z_{1}}{\left(z_{1}+b\right)} \Gamma\left(z_{1}\right)
$$

where $\Gamma(z)$ is the convolution integral, defined by Eq. (43). By writing $\Gamma(z)$ as:

$$
\Gamma(z)=\int_{0}^{\infty} \mathrm{d} z^{\prime} \Gamma\left(z^{\prime}\right) \delta\left(z-z^{\prime}\right)
$$

$w(z)$ can be rewritten in the following form:

$$
\begin{aligned}
w(z)= & -\frac{1}{z+b}\left\{\frac{b^{3}}{3} \int_{0}^{\infty} \mathrm{d} z^{\prime} \frac{\Gamma\left(z^{\prime}\right)}{z^{\prime}+b}-\frac{1}{3} \int_{0}^{z} \mathrm{~d} z^{\prime}\left(z^{\prime}+b\right)^{2} \Gamma\left(z^{\prime}\right)\right\} \\
& +\frac{(z+b)^{2}}{3} \int_{z}^{\infty} \mathrm{d} z^{\prime} \frac{\Gamma\left(z^{\prime}\right)}{z^{\prime}+b}
\end{aligned}
$$

The effective charge $\sigma_{\text {eff }}$ (or equivalently, the effective Gouy-Chapman length $b_{\text {eff }}$ ) can be calculated from the coefficient of $z^{-1}$ in $w(z)$, as $z$ approaches infinity:

$$
w(z) \sim \frac{2\left(b-b_{\mathrm{eff}}\right)}{z}, \quad z \rightarrow \infty
$$

We thus find:

$$
b_{\text {eff }}-b=\frac{1}{6} \int_{0}^{\infty} \mathrm{d} z\left[\frac{b^{3}}{z+b}-(z+b)^{2}\right] \Gamma(z)
$$

A simple form for the convolution integral $\Gamma(z)$ can be obtained in the limits in which $b$ is small or large relative to $d_{\text {hyd }}$, the characteristic range of the hydration potential.

\section{Slowly varying density: $b \gg d_{\text {hyd }}$}

In the limit $b \gg d_{\text {hyd }}$, the PB distribution varies slowly on the scale of the hydration interaction. The convolution integral $\Gamma(z)$ of Eq. (43) can then be approximated in the following way:

$$
\begin{aligned}
\Gamma(z) & =\frac{1}{2 \pi l_{\mathrm{B}}} \int_{-\infty}^{\infty} \mathrm{d} z^{\prime} \frac{H\left(z^{\prime}\right)}{\left(z^{\prime}+b\right)^{2}} \frac{\mathrm{d}^{2} B}{\mathrm{~d} z^{2}}\left(z-z^{\prime}\right) \\
& =\frac{1}{2 \pi l_{\mathrm{B}}} \int_{-\infty}^{\infty} \mathrm{d} z^{\prime}\left[\frac{1}{b^{2}} \frac{\mathrm{d} \delta(z)}{\mathrm{d} z}-\frac{2}{b^{3}} \delta(z)+\frac{6 H(z)}{(z+b)^{4}}\right] B\left(z-z^{\prime}\right) \\
& \simeq \frac{B_{\mathrm{t}}}{2 \pi l_{\mathrm{B}}} \cdot\left[\frac{1}{b^{2}} \frac{\mathrm{d} \delta(z)}{\mathrm{d} z}-\frac{2}{b^{3}} \delta(z)+\frac{6 H(z)}{(z+b)^{4}}\right]
\end{aligned}
$$

where $H(z)$ is the Heaviside function $(H(z)=0$ for $z<0$ and $\mathrm{H}(\mathrm{z})=1$ for $z>0)$. Inserting this expression in Eq. (B5) we obtain Eq. (45) for the effective Gouy-Chapman length. By substituting equation (B6) in Eq. (B3), the form of $w(z)$, given in Eq. (46), is obtained. 


\section{Approximated form for $B(z)$}

Some of the following results depend on the specific structure of the hydration interaction, characterized by the function $B(z)$. In order to obtain analytical expressions, we use a simple approximated form, $B^{\text {app }}(z)$, instead of $B(z)$. Assuming that the hydration interaction consists of a hard core interaction and a short-range attractive part, the function $B(z)$ has some general characteristics that should be present in $B^{\operatorname{app}}(z)$. For $z<d_{\mathrm{hc}}, B(z)$ always has the parabolic form $-\left(B_{0}+\pi z^{2}\right)$, where $B_{0}=-B(z=0)$. We assume that the attractive part of the interaction dominates over the short-range repulsion so that $B_{0}$ is positive. For $z$ larger than some finite value $d_{\text {hc }}+\Delta, B(z)$ is practically zero due to the short range of the interaction. For $d_{\mathrm{hc}}<z<d_{\mathrm{hc}}+\Delta, B(z)$ varies from $-\left(B_{0}+\pi d_{\mathrm{hc}}^{2}\right)$ to zero in a functional form that depends on the details of the attractive potential. The most simple way to model this behavior of $B(z)$ is to have a linear increase of $B^{\operatorname{app}}(z)$ between $z=d_{\mathrm{hc}}$ and $z=d_{\mathrm{hc}}+\Delta$, and to set $B^{\text {app }}$ to be zero for $z$ larger than $d_{\mathrm{hc}}+\Delta$ :

$$
B^{\operatorname{app}}(z)= \begin{cases}-\left(B_{0}+\pi z^{2}\right) & |z| \leq d_{\mathrm{hc}} \\ -\left(B_{0}+\pi d_{\mathrm{hc}}^{2}\right) \frac{\left(d_{\mathrm{hc}}+\Delta-z\right)}{\Delta} & d_{\mathrm{hc}}<|z| \leq d_{\mathrm{hc}}+\Delta \\ 0 & d_{\mathrm{hc}}+\Delta<|z|\end{cases}
$$

The parameters in this expression should be chosen to match, approximately, the form of $B(z)$. Setting $d_{\mathrm{hc}}$ to be the hard core diameter of the real potential and setting $B_{0}=-B(0)$ ensures that $B(z)$ and $B^{\text {app }}(z)$ are identical for $z<d_{\mathrm{hc}}$. The width $\Delta$ can then be set such that $B_{\mathrm{t}}^{\mathrm{app}}=B_{\mathrm{t}}$ :

$$
2 B_{0} d_{\mathrm{hc}}+\frac{2}{3} d_{\mathrm{hc}}^{3}+\Delta\left(B_{0}+\pi d_{\mathrm{hc}}^{2}\right)=-B_{\mathrm{t}}
$$

This is desirable in light of equations (45) and (48), since the effective surface charge depends only on $B_{\mathrm{t}}$ in these limits. Figure 11 shows $B(z)$ and $B^{\text {app }}(z)$ for the hydration potential of Fig. 2.

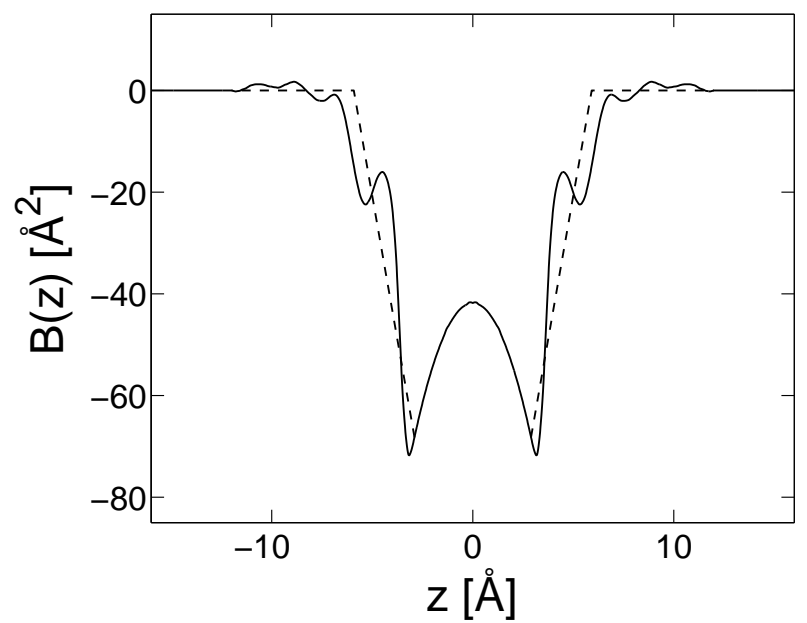

FIG. 11. The effective interaction in a planar geometry, $B(z)$, obtained from the potential of Fig. 2, and the corresponding approximated function $B^{\text {app }}(z)$, defined by Eq. (B7) (dashed line). The parabolic dependance for $|z|<d_{\mathrm{hc}}$ is identical in the two curves.

\section{Surface layer limit: $b \ll d_{\text {hyd }}$}

In the limit $b \ll d_{\text {hyd }}$, the convolution integral in Eq. (43) becomes:

$$
\Gamma(z) \simeq \frac{|\sigma|}{e} \frac{\mathrm{d}^{2} B(z)}{\mathrm{d} z^{2}}=\frac{1}{2 \pi l_{\mathrm{B}} b} \frac{\mathrm{d}^{2} B(z)}{\mathrm{d} z^{2}}
$$

The prefactor of $\Gamma(z)$ in Eq. $(\sqrt{\mathrm{B} 5})$ is $-\frac{1}{6} z^{2}+O(b)$ and therefore the effective Gouy-Chapman length is:

$$
b_{\mathrm{eff}}-b \cong \frac{-1}{12 \pi l_{\mathrm{B}} b} \int_{0}^{\infty} \mathrm{d} z z^{2} B^{\prime \prime}(z)=\frac{-B_{\mathrm{t}}}{12 \pi l_{\mathrm{B}}} \frac{1}{b}
$$


This result is independent on the specific form of $B(z)$.

To obtain $w(z)$, the deviation of the density profile relative to PB theory, Eq. (B9) can be substituted in Eq. (B3). Up to leading order in $b$ the following expression is obtained:

$$
w(z)=\frac{1}{6 \pi l_{\mathrm{B}} b} \frac{1}{z} \int_{0}^{z} \mathrm{~d} z^{\prime} B^{\prime \prime}\left(z^{\prime}\right) z^{\prime 2}+\frac{1}{6 \pi l_{\mathrm{B}} b} z^{2} \int_{z}^{\infty} \mathrm{d} z^{\prime} \frac{1}{z^{\prime}} B^{\prime \prime}\left(z^{\prime}\right)
$$

Using $B^{\text {app }}(z)$, the approximated form of $B(z)$ presented in the previous subsection, this gives:

$$
w(z)=\left\{\begin{array}{cr}
\frac{1}{6 \pi l_{\mathrm{B}} b} z^{2}\left(\frac{4 \pi}{3}+\frac{B_{0}+\pi d_{\mathrm{hc}}^{2}}{d_{\mathrm{hc}}\left(d_{\mathrm{hc}}+\Delta\right)}-2 \pi \log \frac{d_{\mathrm{hc}}}{z}\right), & |z| \leq d_{\mathrm{hc}} \\
\frac{1}{6 \pi l_{\mathrm{B}} b}\left[d_{\mathrm{hc}}^{2}\left(\frac{4 \pi}{3} d_{\mathrm{hc}}+\frac{B_{0}+\pi d_{\mathrm{hc}}^{2}}{\Delta}\right) \frac{1}{z}\right. & d_{\mathrm{hc}}<|z| \leq d_{\mathrm{hc}}+\Delta \\
\left.\frac{B_{0}+\pi d_{\mathrm{hc}}^{2}}{\Delta\left(d_{\mathrm{hc}}+\Delta\right)} z^{2}\right], & d_{\mathrm{hc}}+\Delta<|z| \\
\frac{B_{\mathrm{t}}}{6 \pi l_{\mathrm{B}} b}, &
\end{array}\right.
$$

The minimal, negative value of $w(z)$ is assumed at $z=d_{\mathrm{hc}}+\Delta$ and is equal to:

$$
w\left(d_{\mathrm{hc}}+\Delta\right)=\frac{B_{\mathrm{t}}}{6 \pi l_{\mathrm{B}} b\left(d_{\mathrm{hc}}+\Delta\right)} \simeq \frac{B_{\mathrm{t}}}{6 \pi l_{\mathrm{B}} b d_{\mathrm{hyd}}}
$$

This results in the condition (49) for the validity of the linearization in the surface layer limit.

Using only the quadratic term in the virial expansion is sensible if $\int_{0}^{\infty} \mathrm{d} z^{\prime} c\left(z^{\prime}\right) B\left(z-z^{\prime}\right)$ is small compared to unity. In the surface layer limit, this integral is simply: $(|\sigma| / e) B(z)=B(z) /\left(2 \pi l_{\mathrm{B}} b\right)$. Estimating the maximum value of $|B(z)|$ to be approximately $-B_{\mathrm{t}} /\left(2 d_{\mathrm{hyd}}\right)$ we obtain the requirement: $-B_{\mathrm{t}} /\left(4 \pi l_{\mathrm{B}} b d_{\mathrm{hyd}}\right) \ll 1$, which is analogous to (49).

\section{Effective Gouy-Chapman length}

Using $B^{\operatorname{app}}(z)$ in equations (43) and $(\mathrm{B} 5)$ we find the following approximation for the effective Gouy-Chapman length:

$$
\begin{aligned}
b_{\mathrm{eff}}-b= & \frac{1}{12 \pi l_{\mathrm{B}} b}\left\{-B_{\mathrm{t}}^{\mathrm{app}}-\pi d_{\mathrm{hc}}^{2} b+2 \pi d_{\mathrm{hc}} b^{2}+2 B_{0} \log \left(\frac{b+d_{\mathrm{hc}}+\Delta}{b}\right) b\right. \\
& -\frac{2}{\Delta}\left(\pi d_{\mathrm{hc}}^{2} \Delta+\pi d_{\mathrm{hc}}^{3}+B_{0} d_{\mathrm{hc}}\right) \log \left(\frac{b+d_{\mathrm{hc}}}{b+d_{\mathrm{hc}}+\Delta}\right) b \\
& \left.-2 \pi \log \left(\frac{b+d_{\mathrm{hc}}}{b}\right) b^{3}\right\}
\end{aligned}
$$

This expression is shown in Fig. 10 and discussed in section IV. In the limits $b \gg d_{\text {hyd }}$ and $b \ll d_{\text {hyd }}$ it reduces to the asymptotic expressions (45) and 48), respectively.

[1] G. Gouy, J. Phys. (France) 9, 457 (1910).

[2] D. L. Chapman, Philos. Mag. 25, 475 (1913).

[3] P. Debye and E. Hückel, Phyzik 24, 185 (1923).

[4] P. Debye and E. Hückel, Phyzik 25, 97 (1924).

[5] J. Israelachvili, Intermolecular and Surface Forces, 2nd ed. (Academic Press Inc., New York, 1991).
[6] D. Andelman, in Handbook of Physics of Biological Systems, edited by R. Lipowsky and E. Sackmann (Elsevier Science, Amsterdam, 1994), Vol. I, Chap. 12, p. 603.

[7] B. Honig and A. Nicholls, Science 268, 1144 (1995).

[8] E. J. W. Verwey and J. T. G. Overbeek, Theory of the Stability of Lyophobic Colloids (Elsevier, Amsterdam, 1948).

[9] S. Alexander et al., J. Chem. Phys 80, 5776 (1984).

[10] J. L. Barrat and J. F. Joanny, Adv. Chem. Phys. XCIV, 1 (1996). 
[11] D. Harries, S. May, W. M. Gelbart, and A. Ben-Shaul, Biophys. J. 75, 159 (1998).

[12] I. Borukhov, D. Andelman, and H. Orland, Macromolecules 31, 1665 (1998).

[13] G. J. Fleer et al., Polymers at Interfaces (Chapman \& Hall, London, 1993).

[14] R. Kjellander and S. Marčelja, J. Chem. Phys. 82, 2122 (1985).

[15] R. Kjellander, J. Chem. Phys. 88, 7129 (1988).

[16] S. Levine, C. W. Outhwaite, and L. B. Bhuiyan, J. Electroanal. Chem. 123, 105 (1981).

[17] C. W. Outhwaite and L. B. Bhuiyan, J. Chem. Soc. Faraday Trans. 2 79, 707 (1983).

[18] L. Blum and D. Henderson, in Fundamentals of Inhomogeneous Fluids, edited by D. Henderson (Marcel Dekker, Inc., New York, 1992), Chap. 6, pp. 239-276, and references therein.

[19] R. R. Netz and H. Orland, Europhys. Lett. 45, 726 (1999).

[20] R. R. Netz and H. Orland, Europhys. J. E. 1, 203 (2000).

[21] L. Guldbrand, B. Jönsson, H. Wennerström, and P. Linse, J. Chem. Phys. 80, 2221 (1984).

[22] R. Kjellander, T. Åkesson, B. Jönsson, and S. Marčelja, J. Chem. Phys. 97, 1424 (1992).

[23] H. Greberg, R. Kjellander, and T. Åkesson, Mol. Phys. 92, 35 (1997).

[24] M. J. Stevens and M. O. Robbins, Europhys. Lett. 12, 81 (1990).

[25] I. Borukhov, D. Andelman, and H. Orland, Phys. Rev. Lett. 79, 435 (1997).

[26] I. Borukhov, D. Andelman, and H. Orland, Electrochim. Acta (in press).

[27] D. B. Lukatsky and S. A. Safran, Phys. Rev. E. 60, 5848 (1999).

[28] G. M. Torrie and G. N. Patey, Electrochim. Acta 36, 1677 (1991).

[29] J. Israelachvili and H. Wennerström, Nature 379, 219 (1996).

[30] F. Otto and G. N. Patey, Phys. Rev. Lett. 60, 4416
(1999).

[31] R. M. Pashley, J. Colloid Interface Sci. 80, 153 (1981).

[32] R. M. Pashley and J. N. Israelachvili, J. Colloid Interface Sci. 101, 511 (1984).

[33] B. V. Derjaguin and L. D. Landau, Acta Phys. Chem. USSR XIV, 633 (1941).

[34] S. Marčelja, Nature 385, 689 (1997).

[35] S. Marčelja, Langmuir (in press).

[36] E. Guàrdia, R. Rey, and J. A. Padró, J. Chem. Phys. 95, 2823 (1991).

[37] E. Guàrdia, R. Rey, and J. A. Padró, Chem. Phys. 155, 187 (1991).

[38] D. E. Smith and L. X. Dang, J. Chem. Phys. 100, 3757 (1994).

[39] A. P. Lyubartsev and A. Laaksonen, Phys. Rev. Lett. 55, 5689 (1997).

[40] A. P. Lyubartsev and A. Laaksonen, Phys. Rev. Lett. 52, 3730 (1995).

[41] S. Marčelja, Period. Biol. 100, 7 (1998).

[42] S. Marčelja, Colloid Surface A 130, 321 (1997).

[43] Y. Burak and D. Andelman, submitted to J. Chem. Phys.

[44] S. Marčelja, private communication.

[45] L. Lue, N. Zoeller, and D. Blankschtein, Langmuir 15, 3726 (1999).

[46] J. K. Percus, J. Stat. Phys. 42, 921 (1986).

[47] L. D. Landau and E. M. Lifshitz, Statistical Physics, 3rd edition, Part 1 (Pergamon Press, New York, 1980).

[48] We are indebted to R. Netz for raising this point.

[49] An ion-surface interaction is treated on a mean-field level in: M. N. Tamashiro and P. Pincus, Phys. Rev. E 60, 6549 (1999)

[50] S. L. Carnie and D. Y. C. Chan, J. Chem. Phys. 74, 1293 (1981).

[51] R. Kjellander, Ber. Bunsenges. Phys. Chem. 100, 894 (1996).

[52] G. S. Manning, J. Chem. Phys. 51, 954 (1969).

[53] T. Morita and K. Hiroike, Prog. Theor. Phys. 25, 537 (1961). 